\title{
Evidence for a Supergalactic Structure of Magnetic Deflection Multiplets of Ultra-high-energy Cosmic Rays
}

\author{
Rasha U. Abbasi \\ Loyola University Chicago, rabbasi@luc.edu
}

Follow this and additional works at: https://ecommons.luc.edu/physics_facpubs

Part of the Physics Commons

\section{Recommended Citation}

Abbasi, Rasha U., "Evidence for a Supergalactic Structure of Magnetic Deflection Multiplets of Ultra-highenergy Cosmic Rays" (2020). Physics: Faculty Publications and Other Works. 65.

https://ecommons.luc.edu/physics_facpubs/65

This Article is brought to you for free and open access by the Faculty Publications and Other Works by Department at Loyola eCommons. It has been accepted for inclusion in Physics: Faculty Publications and Other Works by an authorized administrator of Loyola eCommons. For more information, please contact ecommons@luc.edu. (c) AAS, 2020. Reproduced with permission. All rights reserved 


\title{
Evidence for a Supergalactic Structure of Magnetic Deflection Multiplets of Ultra-high- energy Cosmic Rays
}

R. U. Abbasi ${ }^{1}$ (1) , M. Abe , T. Abu-Zayyad $^{3}$ (1) , M. Allen ${ }^{3}$, R. Azuma ${ }^{4}$, E. Barcikowski ${ }^{3}$, J. W. Belz ${ }^{3}$, D. R. Bergman ${ }^{3}$, S. A. Blake ${ }^{3}$, R. Cady $^{3}$, B. G. Cheon ${ }^{5}$, J. Chiba ${ }^{6}$, M. Chikawa ${ }^{7}$, A. di Matteo ${ }^{8,37}$ (i), T. Fujii ${ }^{9}$ (i) K. Fujisue ${ }^{7}$, K. Fujita ${ }^{10}$, R. Fujiwara ${ }^{10}$,

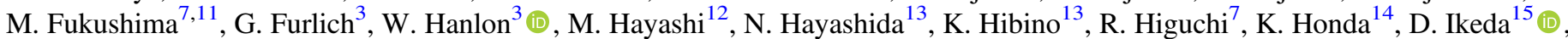
T. Inadomi ${ }^{16}$, N. Inoue ${ }^{2}$, T. Ishii ${ }^{14}$, R. Ishimori ${ }^{4}$, H. Ito ${ }^{17}$, D. Ivanov ${ }^{3}$ (1), H. Iwakura ${ }^{16}$, H. M. Jeong ${ }^{18}$, S. Jeong ${ }^{18}$, C. C. H. Jui ${ }^{3}$ (1), K. Kadota ${ }^{19}$, F. Kakimoto ${ }^{13}$, O. Kalashev ${ }^{20}$, K. Kasahara ${ }^{21}$, S. Kasami ${ }^{22}$, H. Kawai ${ }^{23}$, S. Kawakami ${ }^{10}$, S. Kawana ${ }^{2}$, K. Kawata ${ }^{7}$, E. Kido ${ }^{7}$, H. B. Kim ${ }^{5}$, J. H. Kim ${ }^{10}$, J. H. Kim ${ }^{3}$, M. H. Kim ${ }^{18}$, S. W. Kim ${ }^{18}$, S. Kishigami ${ }^{10}$, V. Kuzmin ${ }^{20,38}$, M. Kuznetsov ${ }^{8,20}$, Y. J. Kwon ${ }^{24}$, K. H. Lee ${ }^{18}$, B. Lubsandorzhiev ${ }^{20}$, J. P. Lundquist ${ }^{3,25,39}$ (1), K. Machida ${ }^{14}$, H. Matsumiya ${ }^{10}$, T. Matsuyama ${ }^{10}$, J. N. Matthews ${ }^{3}$ (1) R. Mayta ${ }^{10}$, M. Minamino ${ }^{10}$, K. Mukai $^{14}$, I. Myers ${ }^{3}$, S. Nagataki ${ }^{17}$, K. Nakai $^{10}$, R. Nakamura ${ }^{16}$, T. Nakamura $^{26}$, Y. Nakamura ${ }^{16}$, T. Nonaka ${ }^{7}$, H. Oda ${ }^{10}$, S. Ogio ${ }^{10,27}$, M. Ohnishi ${ }^{7}$, H. Ohoka ${ }^{7}$, Y. Oku ${ }^{22}$, T. Okuda ${ }^{28}$, Y. Omura ${ }^{10}$, M. Ono $^{17}$, R. Onogi ${ }^{10}$, A. Oshima ${ }^{10}$, S. Ozawa ${ }^{29}$, I. H. Park ${ }^{18}$, M. S. Pshirkov ${ }^{20,30}$, J. Remington ${ }^{3}$, D. C. Rodriguez ${ }^{3}$, G. Rubtsov ${ }^{20}$ (1), D. Ryu $^{31}$, H. Sagawa ${ }^{7}$, R. Sahara ${ }^{10}$, Y. Saito ${ }^{16}$, N. Sakaki ${ }^{7}$, T. Sako ${ }^{7}$, N. Sakurai ${ }^{10}$, K. Sano ${ }^{16}$, T. Seki ${ }^{16}$, K. Sekino ${ }^{7}$, P. D. Shah ${ }^{3}$, F. Shibata ${ }^{14}$, T. Shibata ${ }^{7}$, H. Shimodaira ${ }^{7}$, B. K. Shin ${ }^{31}$, H. S. Shin $^{7}$, J. D. Smith ${ }^{3}$, P. Sokolsky ${ }^{3}$, N. Sone ${ }^{16}$, B. T. Stokes ${ }^{3}$,

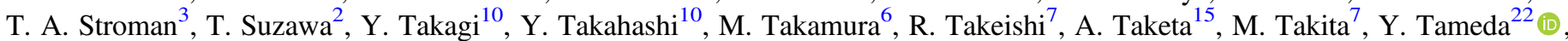
H. Tanaka ${ }^{10}$, K. Tanaka ${ }^{32}$, M. Tanaka ${ }^{33}$, Y. Tanoue ${ }^{10}$, S. B. Thomas ${ }^{3}$, G. B. Thomson ${ }^{3}$, P. Tinyakov ${ }^{8,20}$, I. Tkachev $^{20}$, H. Tokuno $^{4}$, T. Tomida ${ }^{16}$, S. Troitsky ${ }^{20}$ (1), Y. Tsunesada ${ }^{10,27}$ (i), Y. Uchihori ${ }^{34}$, S. Udo ${ }^{13}$, T. Uehama ${ }^{16}$, F. Urban ${ }^{35}$, T. Wong ${ }^{3}$, K. Yada ${ }^{7}$, M. Yamamoto ${ }^{16}$, K. Yamazaki ${ }^{13}$, J. Yang ${ }^{36}$, K. Yashiro ${ }^{6}$, M. Yosei ${ }^{22}$, Y. Zhezher ${ }^{7,20}$, and Z. Zundel ${ }^{3}$

${ }^{1}$ Department of Physics, Loyola University Chicago, Chicago, IL, USA

2 The Graduate School of Science and Engineering, Saitama University, Saitama, Saitama, Japan
${ }^{3}$ High Energy Astrophysics Institute and Department of Physics and Astronomy, University of Utah, Salt Lake City, Utah, USA; jplundquist@gmail.com

${ }^{4}$ Graduate School of Science and Engineering, Tokyo Institute of Technology, Meguro, Tokyo, Japan

${ }^{5}$ Department of Physics and The Research Institute of Natural Science, Hanyang University, Seongdong-gu, Seoul, Republic of Korea ${ }^{6}$ Department of Physics, Tokyo University of Science, Noda, Chiba, Japan

${ }_{8}^{7}$ Institute for Cosmic Ray Research, University of Tokyo, Kashiwa, Chiba, Japan

${ }^{8}$ Service de Physique Théorique, UniversitéLibre de Bruxelles, Brussels, Belgium

${ }^{9}$ The Hakubi Center for Advanced Research and Graduate School of Science, Kyoto University, Kitashirakawa-Oiwakecho, Sakyo-ku, Kyoto, Japan ${ }^{10}$ Graduate School of Science, Osaka City University, Osaka, Osaka, Japan

${ }^{11}$ Kavli Institute for the Physics and Mathematics of the Universe (WPI), Todai Institutes for Advanced Study, University of Tokyo, Kashiwa, Chiba, Japan

${ }^{12}$ Information Engineering Graduate School of Science and Technology, Shinshu University, Nagano, Nagano, Japan

${ }^{13}$ Faculty of Engineering, Kanagawa University, Yokohama, Kanagawa, Japan

${ }^{14}$ Interdisciplinary Graduate School of Medicine and Engineering, University of Yamanashi, Kofu, Yamanashi, Japan

${ }^{15}$ Earthquake Research Institute, University of Tokyo, Bunkyo-ku, Tokyo, Japan

${ }^{16}$ Academic Assembly School of Science and Technology Institute of Engineering, Shinshu University, Nagano, Nagano, Japan ${ }^{17}$ Astrophysical Big Bang Laboratory, RIKEN, Wako, Saitama, Japan

${ }^{18}$ Department of Physics, Sungkyunkwan University, Jang-an-gu, Suwon, Republic of Korea

${ }^{19}$ Department of Physics, Tokyo City University, Setagaya-ku, Tokyo, Japan

${ }^{20}$ Institute for Nuclear Research of the Russian Academy of Sciences, Moscow, Russia

${ }^{21}$ Faculty of Systems Engineering and Science, Shibaura Institute of Technology, Minato-ku, Tokyo, Japan

${ }^{22}$ Department of Engineering Science, Faculty of Engineering, Osaka Electro-Communication University, Neyagawa-shi, Osaka, Japan

${ }^{23}$ Department of Physics, Chiba University, Chiba, Chiba, Japan

${ }^{24}$ Department of Physics, Yonsei University, Seodaemun-gu, Seoul, Republic of Korea

${ }^{25}$ Center for Astrophysics and Cosmology, University of Nova Gorica, Ajdovščina, Slovenia ${ }^{26}$ Faculty of Science, Kochi University, Kochi, Kochi, Japan

${ }^{27}$ Nambu Yoichiro Institute of Theoretical and Experimental Physics, Osaka City University, Osaka, Osaka, Japan

${ }^{28}$ Department of Physical Sciences, Ritsumeikan University, Kusatsu, Shiga, Japan

${ }^{29}$ Quantum ICT Advanced Development Center, National Institute for Information and Communications Technology, Koganei, Tokyo, Japan

${ }^{30}$ Sternberg Astronomical Institute, Moscow M.V. Lomonosov State University, Moscow, Russia

${ }^{31}$ Department of Physics, School of Natural Sciences, Ulsan National Institute of Science and Technology, UNIST-gil, Ulsan, Republic of Korea

${ }^{32}$ Graduate School of Information Sciences, Hiroshima City University, Hiroshima, Hiroshima, Japan ${ }^{33}$ Institute of Particle and Nuclear Studies, KEK, Tsukuba, Ibaraki, Japan

${ }^{34}$ Department of Research Planning and Promotion, Quantum Medical Science Directorate, National Institutes for Quantum and Radiological Science and Technology, Chiba, Chiba, Japan

${ }^{35}$ CEICO, Institute of Physics, Czech Academy of Sciences, Prague, Czech Republic

${ }^{36}$ Department of Physics and Institute for the Early Universe, Ewha Womans University, Seodaaemun-gu, Seoul, Republic of Korea Received 2020 May 14; revised 2020 July 1; accepted 2020 July 1; published 2020 August 14

\begin{abstract}
Evidence for a large-scale supergalactic cosmic-ray multiplet (arrival directions correlated with energy) structure is reported for ultra-high-energy cosmic-ray (UHECR) energies above $10^{19} \mathrm{eV}$ using 7 years of data from the
\end{abstract}

\footnotetext{
37 Currently at INFN, sezione di Torino, Turin, Italy.

${ }^{38}$ Deceased.

39 Corresponding author.
} 
Telescope Array (TA) surface detector and updated to 10 years. Previous energy-position correlation studies have made assumptions regarding magnetic field shapes and strength, and UHECR composition. Here the assumption tested is that, because the supergalactic plane is a fit to the average matter density of the local large-scale structure, UHECR sources and intervening extragalactic magnetic fields are correlated with this plane. This supergalactic deflection hypothesis is tested by the entire field-of-view (FOV) behavior of the strength of intermediate-scale energy-angle correlations. These multiplets are measured in spherical cap section bins (wedges) of the FOV to account for coherent and random magnetic fields. The structure found is consistent with supergalactic deflection, the previously published energy spectrum anisotropy results of the TA (the Hotspot and Coldspot), and toy-model simulations of a supergalactic magnetic sheet. The seven year data posttrial significance of this supergalactic structure of multiplets appearing by chance, on an isotropic sky, is found by Monte Carlo simulation to be $4.2 \sigma$. The 10 years of data posttrial significance is $4.1 \sigma$. Furthermore, the starburst galaxy M82 is shown to be a possible source of the TA Hotspot, and an estimate of the supergalactic magnetic field using UHECR measurements is presented.

Unified Astronomy Thesaurus concepts: Extragalactic magnetic fields (507); Ultra-high-energy cosmic radiation (1733); Cosmic rays (329); High energy astrophysics (739); Astrophysical magnetism (102); Cosmic ray astronomy (324); Cosmic ray sources (328)

\section{Introduction}

The supergalactic plane (SGP) is the average matter distribution of the local universe up to a distance of $\sim 200 \mathrm{Mpc}$ (a large percentage of its sources are within the GZK horizon of $100 \mathrm{Mpc}$ ) de Vaucouleurs (1975). Large-scale magnetic fields have been measured between clusters of galaxies, which make up the supergalactic plane, including the Coma Cluster, and a 3 Mpc field between A0399 and A0401 (de Vaucouleurs 1975; Bonafede et al. 2010; Govoni et al. 2019).

It has also been shown that $\sim 90 \%$ of the baryonic mass of the universe is contained between galaxies, of which $\sim 40 \%$ is warm-hot protons outside gas clouds Nicastro et al. (2018). This may support the formation of even larger intragalacticscale magnetic fields (Biermann et al. 1997; Ryu et al. 1998). The presence of large-scale magnetic fields suggest that energydependent deflection of ultra-high-energy cosmic rays (UHECRs) may appear correlated with the SGP.

Previous UHECR energy-position correlation (multiplet) searches for small-scale galactic magnetic deflections have not had significant results (Bretz 2011; Abreu et al. 2012; Aab et al. 2015; Wirtz 2019). These multiplet searches used linear correlations of angular distance versus 1/energy and included scanned parameters chosen by the assumed magnetic field models and compositions. The present analysis uses intermediate-scale energy-position correlations (multiplets) to look for significant large-scale magnetic, and source, structure with minimal assumptions regarding particular magnetic field models or UHECR composition.

In this paper the oversampled multiplets are found at grid points evenly covering the field-of-view (FOV) sky, each having their own parameters of size, shape, pointing direction, and energy threshold. The structure of these multiplets is consistent with supergalactic deflection, the previously published energy spectrum anisotropy results of the Telescope Array (TA; the Hotspot and Coldspot; Abbasi et al. 2018a), and toy-model simulations of a supergalactic magnetic sheet (Biermann et al. 1997). Here we report the significance using 7 years of TA data (as in Lundquist \& Sokolsky 2019) and update it to 10 years of data.

\section{Energy-Angle Correlations}

It is assumed that UHECRs are deflected as they travel through coherent magnetic fields according to Equation 1(a), with a deflection variance by random fields as approximated by Equation 1 (b) ( $Z$ is mass number, $B$ is field strength, $S$ is distance traveled in the field, $E$ is particle energy, and $L_{c}$ is mean magnetic field coherence length). These deflection equations are from Roulet (2004) in units more relevant to the extragalactic case. The end effect of these fields is that lower energy cosmic-ray events are deflected to larger angular distances from their source than higher energy events in both lateral and transverse directions Roulet (2004). This driftdiffusion process is diagrammed in Figure 1:

$$
\begin{gathered}
\delta \approx 0.5 Z \frac{B}{\mathrm{nG}} \frac{S}{\mathrm{Mpc}} \frac{10^{20} \mathrm{eV}}{E}, \\
\delta_{\mathrm{rms}} \approx 0.4 Z \frac{B_{\mathrm{rms}}}{\mathrm{nG}} \frac{10^{20} \mathrm{eV}}{E} \sqrt{\frac{S}{\mathrm{Mpc}}} \sqrt{\frac{L_{c}}{\mathrm{Mpc}}} .
\end{gathered}
$$

\subsection{Correlation}

The distance between two points on the surface of a sphere, the great circle angular distance, is shown in Equation (2) in terms of vectors normal to the FOV. Correlations between event energy and angular distance from a grid point are found using a ranked correlation, Kendall's $\tau$, that measures the strength of monotonic dependence Kendall (1945),

$$
\delta_{i j}=\arctan \frac{\left|\hat{\boldsymbol{n}}_{\boldsymbol{i}} \times \hat{\boldsymbol{n}}_{j}\right|}{\hat{\boldsymbol{n}}_{\boldsymbol{i}} \cdot \hat{\boldsymbol{n}}_{\boldsymbol{j}}} .
$$

The Kendall correlation is generally more robust against noise than the other common ranked correlation-Spearman's $\rho$ (Croux \& Dehon 2010). Ranked correlation minimizes the effects on correlation strength by magnetic model (such as higher order terms of Equation 1(a)), composition assumption, energy reconstruction systematics, and detector exposure variation.

Kendall's $\tau$ ranked correlation is the linear correlation between the separate ordering of the two variables of interest (variable $x$ sorted ranks: first, second, third, etc. versus variable $y$ ranks: fifth, first, fourth, etc.), with $n$ pairs of values, and is shown in Equation (3):

$$
\tau=\frac{2}{n(n-1)} \sum_{j<k} \operatorname{sign}\left[\frac{x_{j}-x_{k}}{y_{j}-y_{k}}\right] .
$$

This correlation can be considered simply as the normalized sum of the sign of the slopes between all pairs of data points. A 


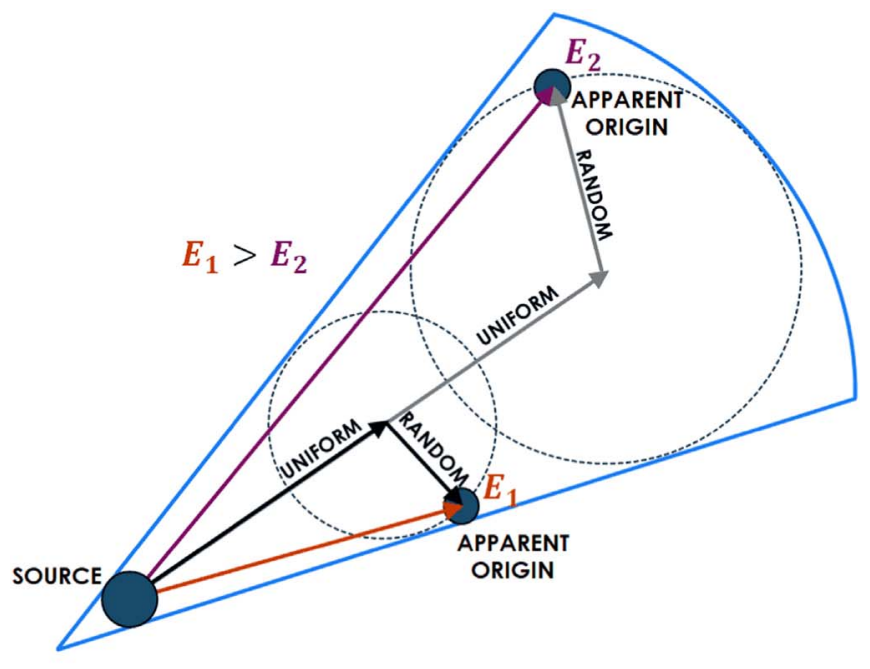

(a)

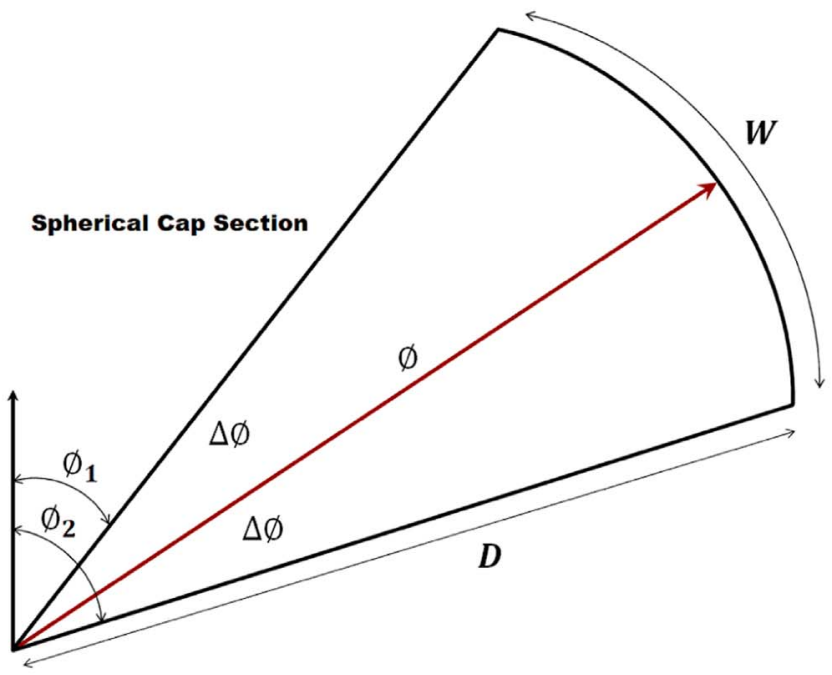

(b)

Figure 1. Pictograph of UHECR drift-diffusion deflection "wedge" bins (spherical cap sections) displayed on a flat space. (a) Two different energy events having traveled through coherent and random magnetic fields. The purple vector represents the low energy event spherical arc, and the red vector is a higher energy event. Coherent and random magnetic field components describe the average perpendicular to the FOV sphere. Dashed circles represent possible random field rms deflections. (b) A spherical cap section (wedge) is a simple shape that best encompasses the likeliest positions. Pointing direction is the spherical arc $\phi, \Delta \phi$ is the wedge width, and $D$ is the maximum angular distance (spherical cap radius).

small correction is made for the rare occurrence of duplicate values and can be found in Kendall (1945).

The correlation coefficient $\tau$ has a range from -1 to +1 , and a value of 0 means that there is no measured relationship between the variables. For +1 , an increase (decrease) of $x$ always follows an increase (decrease) of $y$. If $\tau=-1$ an increase (decrease) of $x$ always follows a decrease (increase) in $y$ (in this analysis, $x$ and $y$ are energy and angular distance). A negative correlation is consistent with the expectation for magnetic field deflected events-as energy decreases, deflection increases, as can be seen from Equation (1).

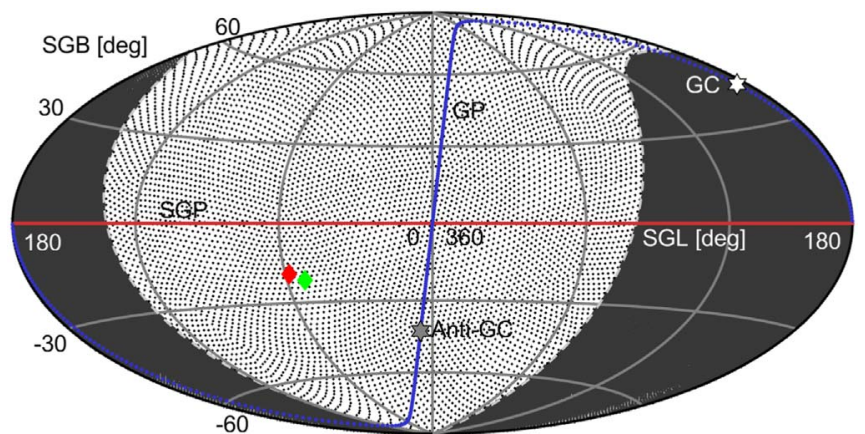

Figure 2. A supergalactic Hammer-Aitoff projection of the equal distance oversampling grid. This is a grid of 6553 points with a mean spacing of $2: 1 \pm 0.1$. The grid boundary is defined by the equatorial edge of the FOV at decl. $=-16^{\circ}$. The red diamond is the location of the Hotspot (Abbasi et al. 2014), and the green diamond is the location of the energy spectrum anisotropy (Abbasi et al. 2018a). The red line is the supergalactic plane (SGP), and the blue line is the galactic plane (GP).

Any monotonic function $\left(x^{b}, \log _{10}(x), e^{x}\right.$, etc.) of distance, energy, or both will always return a $\tau$ coefficient with the same magnitude but not necessarily the same sign $( \pm)$. The sign of the resulting $\tau$ would be the original $\tau$ multiplied by the signs of the first derivatives of the applied functions.

The pretrial two-sided significance of a correlation, $z$ (probability of $\tau=0$ ), is a function of correlation strength and sample size $n$. This significance is found by counting permutations of the sample ranks with greater $\tau$, or in the large $n$ sample limit, Equation (4) (for $n \geqslant 50$ ), which follows the standard normal distribution. Further details can be found in Kendall (1945),

$$
z=\frac{\tau 3 n(n-1) / 2}{\sqrt{n(n-1)(2 n+5) / 2}} .
$$

\subsection{Correlation Binning}

With the drift-diffusion picture of Figure 1 in mind, possible UHECR deflections from grid-point "sources" were found by a scanned maximization of the significance of energy-angle correlations inside spherical cap sections, or "wedges," using seven years of TA data (Lundquist \& Sokolsky 2019). This scan was done at each point on an approximately equal $2^{\circ}$ spaced grid of 6553 points on the FOV shown in Figure 2 (Teanby 2006).

These wedge bins are defined by a maximum angular distance $\delta_{j}$ from the grid point, $i$, defined by Equation (2) and the boundaries of two azimuths defined by Equation (5), where $B$ is latitude and $L$ is longitude:

$$
\phi_{i j}=\arctan \frac{\cos B_{i} \sin \left(L_{i}-L_{j}\right)}{\cos B_{j} \sin B_{i}-\sin B_{j} \cos B_{i} \cos \left(L_{i}-L_{j}\right)} .
$$

The azimuths increase clockwise, and a great circle section, or wedge, pointed toward $90^{\circ}$ supergalactic latitude (SGB) has an azimuth, $\phi$, of $0^{\circ}$, while one pointed toward $-90^{\circ} \mathrm{SGB}$ has a $\phi$ of $180^{\circ}$. The azimuthal angle difference, $\Delta \phi_{i j}$, between the wedge pointing direction, $\phi_{i}$, and the azimuth of an event, $\phi_{i j}$, is shown in Equation (6). An example wedge is shown in Figure 3(a):

$$
\Delta \phi_{i j}=\bmod \left(\left|\phi_{i j}-\phi_{i}\right|+180,360\right)-180
$$

This oversampling bin shape means that four parameters must be scanned at every grid point to maximize the pretrial 


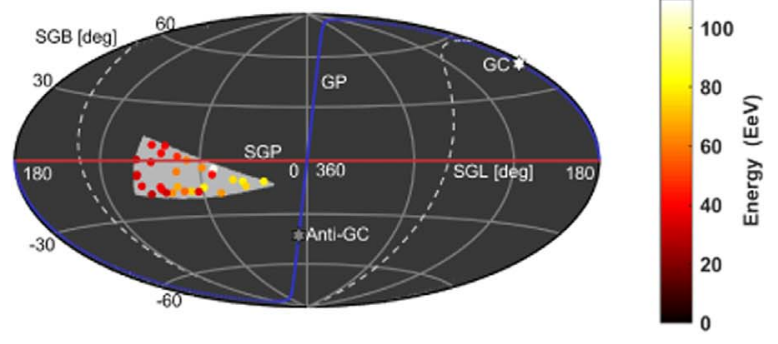

(a)

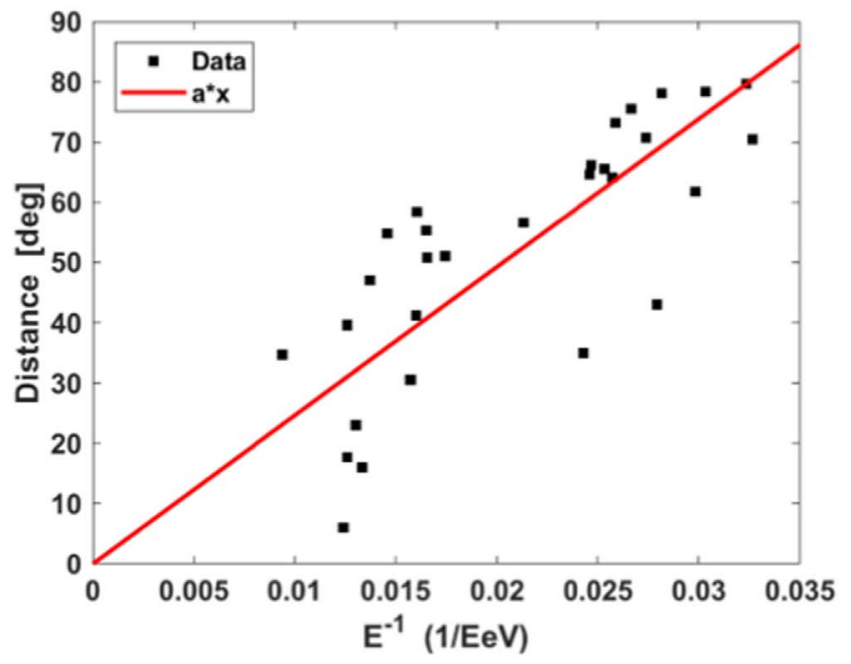

(b)

Figure 3. (a) A supergalactic Hammer-Aitoff projection of the seven year data spherical cap section, or "wedge," with the maximum significance at 18.3 SGB, -12.9 SGL. The correlation strength is $\tau=-0.675$, and with 29 data events has a pretrial one-sided significance of $5.5 \sigma$. The energy threshold is $E_{i} \geqslant 30 \mathrm{EeV}$, wedge width $W_{i}=30^{\circ}$, distance $D_{i}=80^{\circ}$, and direction $\phi_{i}=90^{\circ}$. (b) A scatter plot of $1 / E_{j}$ vs. distance $\delta_{i j}$ for events in the wedge. A linear fit (by Equation 1 (a) with $Z=1$ ) results in an estimate of $B \times S=49 \mathrm{nG} * \mathrm{Mpc}$. If the source is assumed to be the same distance as M82 (3.7 Mpc) with a pure proton emission, then the coherent magnetic field required to cause this deflection would be $B=13 \mathrm{nG}$.

correlation significance. Even though negative correlations are physically expected by a magnetic field drift-diffusion process, both the sign of the correlation, and its strength are not explicitly scanned for nor restricted. The limits on these parameters are large to account for most conceivable extragalactic magnetic deflection scenarios. The scans are all combinations of the following:

1. Energy threshold, $E_{i}: 10$ to $80 \mathrm{EeV}$ in $5 \mathrm{EeV}$ steps.

2. Wedge distance, $D_{i}=\max \left(\delta_{i j}\right): 15^{\circ}$ to $90^{\circ}$ in steps of $5^{\circ}$.

3. Wedge direction, $\phi_{i}: 0^{\circ}$ to $355^{\circ}, 5^{\circ}$ steps.

4. Wedge width, $W_{i}=2^{*} \max \left(\left|\Delta \phi_{i j}\right|\right): 10^{\circ}$ to $90^{\circ}, 10^{\circ}$ steps $\left(5^{\circ}\right.$ on each side of $\phi_{i}$ ).

Events are inside the wedge if $E_{j} \geqslant E_{i}$ and $\delta_{i j} \leqslant D_{i}$ and $-W_{i} / 2 \leqslant \Delta \phi_{i j} \leqslant W_{i} / 2$, where $i$ is the index of the grid point. The energy - angle correlation is calculated inside the wedge, $\tau\left(\delta_{i j}, E_{j}\right)$, and the parameters $\left(E_{i}, D_{i}, \phi_{i}\right.$, and $\left.W_{i}\right)$ are chosen such that the correlation has the minimum $p$-value (Equation (4)). This scan was done using seven years of data. The same bin parameters at each grid point were used for the 10 year data set to test the result.

\subsection{Correlation Example}

The wedge parameters needed to maximize the correlation significance at each grid point were scanned for using seven years of TA data (Lundquist \& Sokolsky 2019). For the seven year data set, the supergalactic coordinates of the most significant correlation of all the grid points is 18.3 SGB (latitude), $-12.9 \mathrm{SGL}$ (longitude). This wedge and the events inside are shown in Figure 3(a). There are 29 events with energies $E \geqslant 30 \mathrm{EeV}$. The pretrial one-sided significance of $\tau=-0.675$ with a sample size of 29 events is $5.5 \sigma$. A scatter plot of energy versus angular distance from the grid point within this wedge is shown in Figure 3(b). A linear fit (Equation 1(a) with $Z=1$ ) results in an estimate of $B \times S=49 \mathrm{nG} * \mathrm{Mpc}$. If the source is assumed to be at the distance to M82 (3.7 Mpc) with a pure proton emission, the average coherent magnetic field, perpendicular to the FOV, required to cause this deflection would be $B=13 \mathrm{nG}$.

Note, however, that the posttrial significance of any single correlation is not expected to be large, as the wedge scan parameter space is large. An individual correlation is not the test of a supergalactic structure.

\section{Simulations}

The same analysis is applied to isotropic simulations in order to calculate the significance of any anisotropy (as described further in Section 4). This is a simulation of data with the TA Surface Detector (SD) configuration while assuming no specific sources or correlation with the supergalactic (or galactic) plane.

A second simulation is used to demonstrate that the analysis is able to find the hypothesized supergalactic structure. This is a simple toy-model simulation of a supergalactic magnetic sheet that results in an energy-dependent diffusion of events away from the supergalactic plane. This sheet simulation is used to motivate the test statistic that tests the hypothesis of supergalactic sources and magnetic fields; this is further described in Section 4. This simulation can also be used to estimate the average coherent field strength between our galaxy and supergalactic sources.

\subsection{Isotropic Simulation}

Each Monte Carlo (MC), and data event, is defined by their energy, zenith angle, azimuthal angle, and trigger time. The latitude and longitude are defined from the center of the TA SD at $39^{\circ} .3$ Lat., $112^{\circ} .9$ Long. These horizontal coordinates are used to calculate the longitude (SGL) and latitude (SGB) in supergalactic coordinates (Vallado 2007). The MC event sets have a zenith angle distribution of $\mathrm{g}(\theta)=\sin (\theta) \cos (\theta)$ due to the event sampling response of a two-dimensional SD array, a uniform azimuth distribution, and the detection efficiency $\sim 100 \%$ for UHECRs $E \geqslant 10^{19.0} \mathrm{eV}$. The event trigger times are approximated as a uniform distribution of modified Julian 


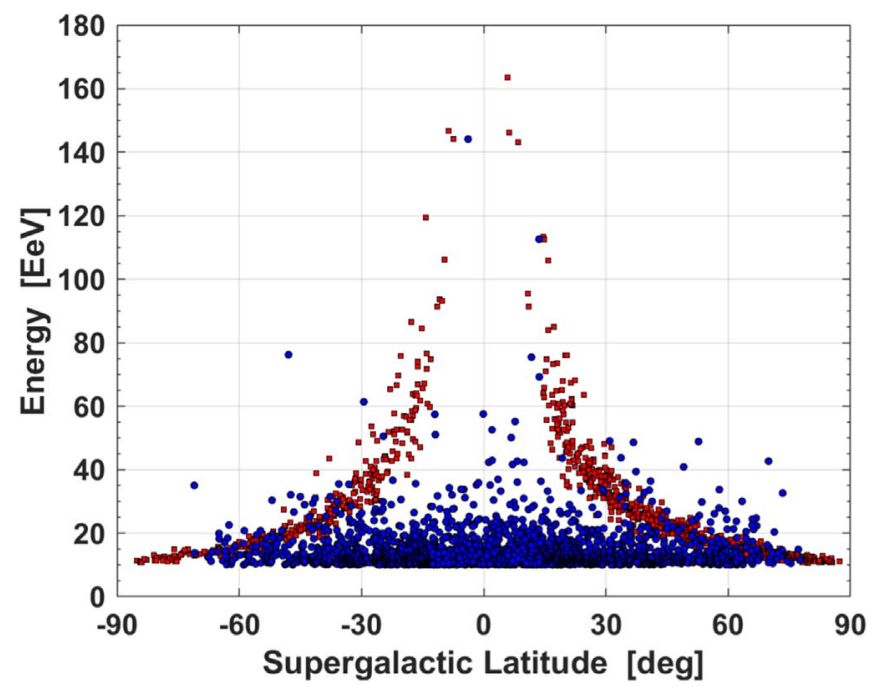

Figure 4. Toy-model supergalactic magnetic sheet simulation. Blue circles are the $F=65.7 \%$ isotropic fraction of MC events. Red squares are the anisotropic MC events magnetically diffused away from the supergalactic plane with $B \times S=18.47 \mathrm{nG} * \mathrm{Mpc}$. Overall, event positions are isotropic, and the energy spectrum is created according to the published HiRes/TA result.

dates from the beginning to the end of the run time due to the approximately $\sim 100 \%$ SD on-time. All of these MC parameter distributions are in good agreement with the data set described in Section 5.

Detector acceptance and bias, in the energy spectrum, are taken into account by interpolation sampling of a large set of $\mathrm{MC}$ events reconstructed through a surface detector simulation thrown with the average HiRes/TA spectrum (Abbasi et al. 2008). The same cuts applied to the data are applied to these fully simulated events, and there are $\sim 4 \times 10^{5}$ with energies $E \geqslant 10^{19.0} \mathrm{eV}$. The number of events in each isotropic MC event set is the same as the data in each $5 \mathrm{EeV}$ bin of the parameter scan of Section 2.2. This simulated data has been shown to reproduce all measured geometric and photoelectric distributions accurately Ivanov (2012).

The result is that each set of these isotropic MC events simulates the expected data, given the detector configuration and on-time, with no energy anisotropies. These MC sets are used to calculate the posttrial probability of any potential anisotropy signal in the data.

\subsection{Supergalactic Magnetic Sheet}

A simple toy-model simulation of an intervening supergalactic magnetic sheet, between the galaxy and UHECR sources, is made by taking the isotropic event sets of Section 3.1 and embedding event deflections (assigning distance correlated energies) in supergalactic latitude (SGB), proportional to $1 /$ energy, for a fraction of events. The coordinates of the MC events are isotropic and unchanged in the procedure. The approximate apparent deflection from the source of a charged particle in a coherent magnetic field is from Equation 1(a). An example of the resulting simulation is shown in Figure 4.

The event deflections, $\delta_{B}$, from supergalactic latitude $\mathrm{SGB}=0^{\circ}$ are calculated for each MC event energy in the set, assuming a proton composition $(Z=1)$ and a particular $B \times S$, according to Equation 1(a). Additionally, some random field noise was added by smearing the $\delta_{B}$ with a $5^{\circ}$ standard deviation Gaussian. Then each event is assigned an energy based on its angular distance from the supergalactic plane (min $\left.\left[\delta_{B}-\delta_{\mathrm{SGB}=0}\right]\right)$. The beginning, and final, simulation is isotropic with respect to the supergalactic longitude (SGL).

After the assignment of an energy to each event position, those with an assigned position-deflection error greater than $10^{\circ}$ are added to the isotropic proportion. This threshold adds additional random field noise in the simulation. This cut also results in a harder spectrum for the deflected events (red event in Figure 4), i.e., higher energy events on average closer to the supergalactic plane. This supergalactic energy bias is due to the lower number of high energy events, resulting in a better fit to a supergalactic magnetic deflection at higher energies (due to the boundary conditions of the energy spectrum and position isotropy).

A supergalactic sheet simulation, with an $F=65.7 \%$ isotropic fraction and $B \times S=18.47 \mathrm{nG} * \mathrm{Mpc}$, is shown in Figure 4 . These parameters are the result of selecting a random MC that looks similar to the data result and the choice of a proton composition. Note again that this is only an anisotropy of energies in supergalactic latitude as event positions are isotropic, and the total energy spectrum is unchanged.

The intent of this toy-model simulation is to show that the analysis method is sensitive to an energy symmetry caused by some kind of magnetic deflection structure correlated with the supergalactic plane. It is not intended to reproduce all aspects of actual data.

\section{Supergalactic Structure}

No single correlation tests the hypothesis that sources and magnetic fields have a relation to local large-scale structure. And no single correlation can be significant when taking into account the average $\sim 60,000$ scan parameter combinations at all 6553 grid points.

As an example of what can be expected, large-scale behavior is demonstrated by the oversampled wedge correlation result for the supergalactic sheet simulation shown in Figure 5(a) ( $F=65.7 \%$ isotropy and $B \times S=18.47 \mathrm{nG} * \mathrm{Mpc})$.

It can be seen via this simple model in the projection of $\tau$ that if there are magnetically induced energy-angle correlations clustered in the supergalactic plane, negative correlation wedges will be close to the supergalactic plane. Furthermore, because negative correlations viewed from the opposite direction appear as positive correlations (as can been seen by Equation (3) for $[x=E, y=D] \rightarrow[x=E, y=-D]$ ), positive correlations are expected at large distances from the supergalactic plane.

\subsection{Significance Test}

Though a test for a supergalactic structure of energy-angle correlations is not necessarily a priori obvious, the supergalactic sheet toy model leads to a reasonable answer. The mean $\langle\tau\rangle$ inside equal solid angle bins of angular distance $\left(\mathrm{SGB}_{i}\right)$ from the supergalactic plane (SGP) shows that three features are relevant for the supergalactic hypothesis-the minimum average $\tau$, the minimum location being near the SGP, and the symmetry of $\tau$ around the SGP. Using all three features to calculate the data significance would be overfitting the problem. One test statistic is preferable though it should be correlated with these three supergalactic structure features. The single parameter chosen to test the supergalactic structure 


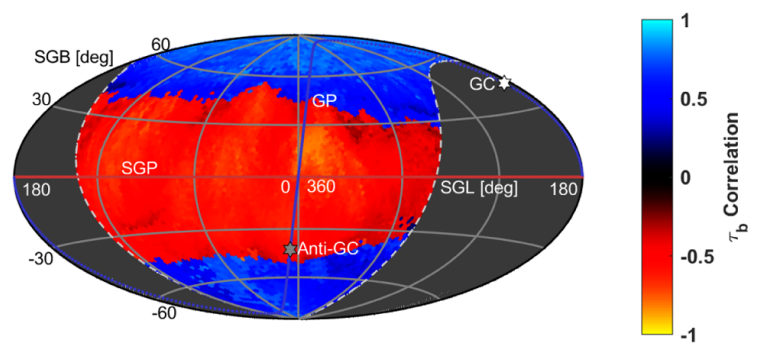

(a)

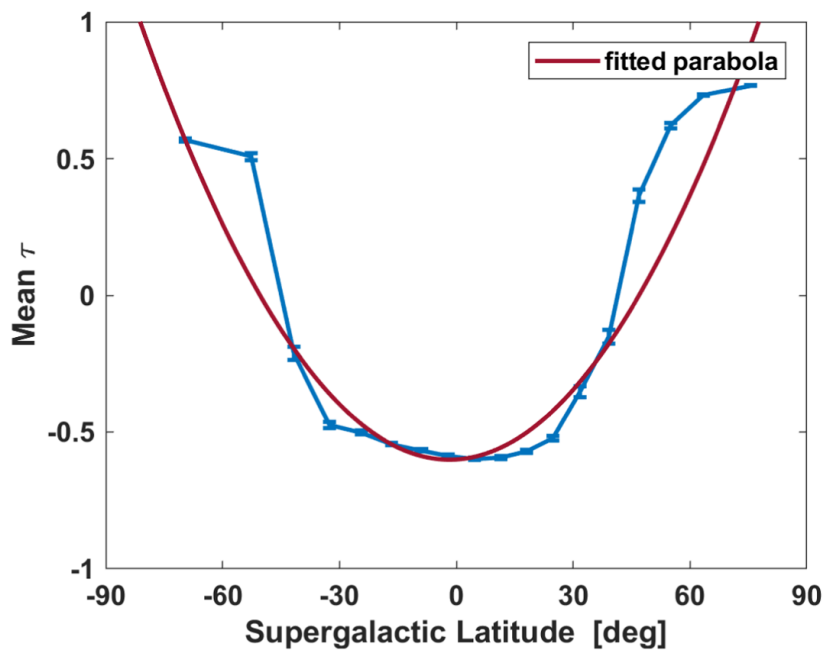

(b)

Figure 5. A supergalactic magnetic sheet simulation. (a) Projection of the correlation strength $\tau$ for all grid points. Solid curves indicate the galactic plane (GP) in blue and supergalactic plane (SGP) in red. (b) Mean $\tau$ inside equal solid angle bins of supergalactic latitude (SGB). The parabolic fit $\left(y=a\left(x-x_{0}\right)^{2}+y_{0}\right)$ shows the curvature parameter, $a$, chosen as the test statistic: $a=2.5 \times 10^{-4}$.

hypothesis is the curvature parameter, " $a$," of a parabolic fit $\left(y=a\left(x-x_{0}\right)^{2}+y_{0}\right)$ to the $\langle\tau\rangle$.

The curvature, " $a$," is simply the lowest-order Taylor expansion term that can describe the symmetry around the SGP shown in the simulation (Figure 5(b)). Due to the boundaries of $|\tau| \leqslant 1$ and $|S G B|<90^{\circ}$, a greater correlation curvature, $a$, corresponds to a minimum, $x_{0}$, closer to the supergalactic plane, as shown in Figure 6(a). A larger curvature $a$ also means that the minimum negative correlation averages are greater in magnitude, $y_{0}$, as shown in Figure 6(b). The parabola minimum $y_{0}$ has no correlation with the minimum supergalactic latitude (SGB). These relationships justify the use of the parabola curvature " $a$ " as the single test statistic for a conservative estimate of the significance of supergalactic energy-angle correlations.

The fit on the large-scale behavior of the correlation strength, $\tau$, is used because it is not explicitly scanned for and contains more information by its sign $( \pm)$ than the pretrial significance. The pretrial significance of the correlations is not used in this analysis so that the significance test is independent of the wedge scan for the maximum significance of individual energy-angle correlations.

To calculate the data significance of a supergalactic structure of energy-angle correlations, the analysis described above was applied to the data and the isotropic MC sets. The number of MC sets with a correlation curvature $a$ greater than the data

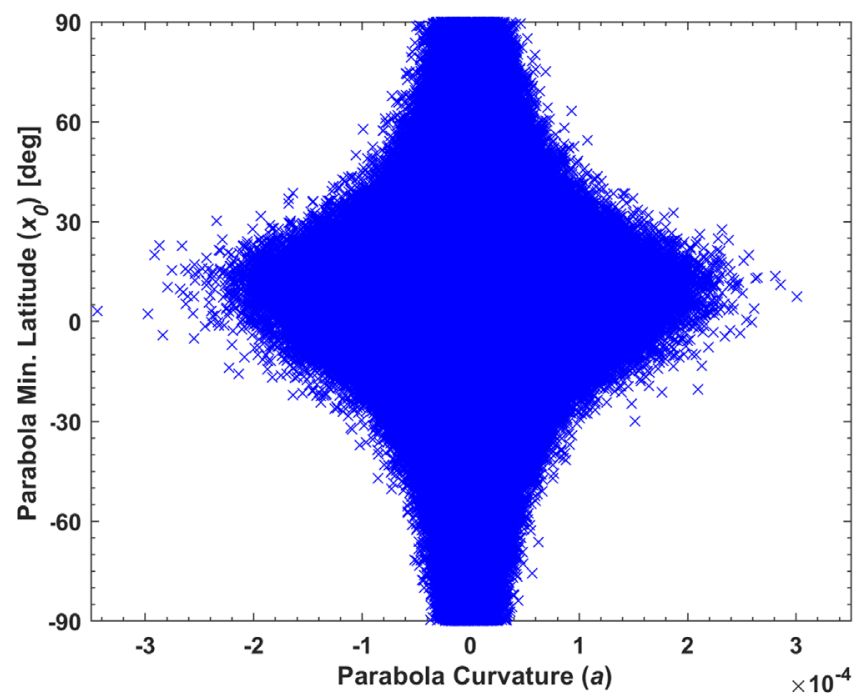

(a)

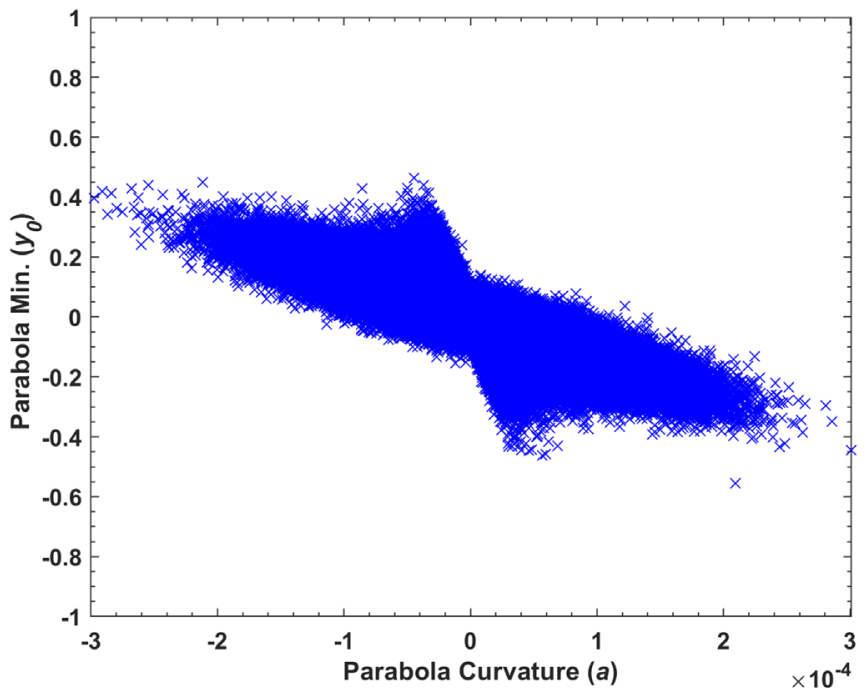

(b)

Figure 6. The behavior of the three mean $\tau$ parabola fit parameters (Figure 5(b)) with respect to each other in random isotropic MC simulations. (a) The parabola fit curvature, $a$, vs. minimum supergalactic latitude (SGB), $x_{0}$, shows that a high curvature tends to a minimum near the supergalactic plane. (b) The parabola fit curvature, $a$, vs. the fit minimum value, $y_{0}$, shows that a high curvature tends to a higher magnitude negative mean $\tau$.

gives the probability of the measured supergalactic structure of energy-angle correlations if there actually is not such a structure, i.e., if it is a statistical fluctuation in the data.

\section{Data Set}

For this analysis, SD data recorded between 2008 and 2019 May 11 are used. Data from 2016 are excluded due to issues with SD communication towers that created a significant dayto-day change of the trigger delay variance within each day of the year. This introduced nonphysical equatorial anisotropies that are nontrivial to compensate for.

The reconstruction method used for these events is the same as the "TA Hotspot" and energy spectrum anisotropy results (Abbasi et al. 2014, 2018a). The energy of reconstructed events is determined by the $\mathrm{SD}$ array and renormalized by $1 / 1.27$ to 
match the calorimetrically determined fluorescence detector energy scale (Abu-Zayyad et al. 2013).

Due to the inclusion of lower energy events, down to $10^{19.0}$ $\mathrm{eV}$, tighter data cuts than the Hotspot analysis are required for good zenith angle and energy resolutions. After cuts, there were 3018 events in the 7 year data set, and there are a total of 4321 events using 10 years of data. Events in the data set match the following criteria:

1. $E \geqslant 10^{19.0} \mathrm{eV}$ (where detection efficiency is $\sim 100 \%$ ).

2. At least four SDs triggered.

3. Zenith angle of arrival direction $<55^{\circ}$.

4. Shower lateral distribution fit $\chi^{2} /$ dof $<10$.

5. Reconstructed pointing direction error $<5^{\circ}$.

6. Shower core $>1.2 \mathrm{~km}$ from array boundary.

The additional cuts on the pointing direction error and boundary distance improve the agreement between the distribution of zenith angles and the geometrical zenith angle distribution $\mathrm{g}(\theta)=\sin (\theta) \cos (\theta)$. The azimuthal angle distribution is in very good agreement with the theoretical uniform distribution. The geometrical zenith angle distribution is due to the flat detector plane, the uniform azimuthal angle distribution, and the detection efficiency of $\sim 100 \%$ for UHECRs with energies $E \geqslant 10^{19.0} \mathrm{eV}$. The energy spectrum is also in good agreement with the published spectrum (Abu-Zayyad et al. 2013; Abbasi et al. 2015). And finally, the event trigger times are in good agreement with the uniform time distribution used for the isotropic MC of Section 3.1.

The energy resolution and pointing direction resolution of events in the data set range from $\sim 10$ to $15 \%$ and $\sim 1{ }^{\circ} 0$ to 1.5 , respectively, depending on core distance from the array boundary and improve with increasing energy. These resolutions are sufficient to search for large-scale and intermediatescale UHECR energy anisotropies.

\section{Results}

The resulting energy-angle correlations for 7 years of data are shown in Figure 7(a) and for 10 years of data is shown in Figure 8(a). Individual correlations with the highest pretrial significance are negative, which means that there is a trend for the angular distance to increase with decreasing energy. This trend is the expectation for a grid point that happens to be near a source of magnetically scattered UHECR events. It can be seen that the negative $\tau$ correlations themselves appear well correlated with the supergalactic plane.

Figure 7(b) shows the seven year data result of the mean $\tau$ correlation inside equal solid angle bins parallel to the supergalactic plane (SGP). The parabolic fit curvature is $a=(2.45 \pm 0.15) \times 10^{-4}$ with a minimum at $-0.5 \mathrm{SGB}$. According to the $R^{2}$ (coefficient of determination) goodness-offit the model predicts $88 \%$ of the variance of the data. The data correlations have a very similar form to that of the supergalactic magnetic sheet simulation, shown in Figure 5(a), that has $a=2.5 \times 10^{-4}$ with a minimum at $-1.7 \mathrm{SGB}$.

Previously, by applying this analysis to isotropic MC sets (using data positions and random energies), the number of $\mathrm{MC}$ with an $a$ parameter greater than data was 2 out of 200,000 trials, which resulted in a posttrial significance of the supergalactic structure of multiplets of $\sim 4 \sigma$ (Lundquist \& Sokolsky 2019).

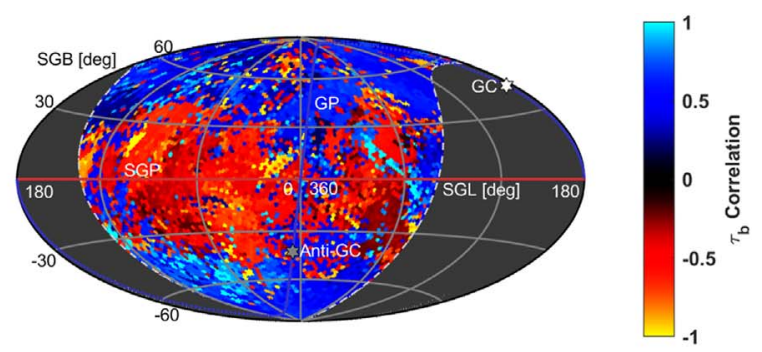

(a)

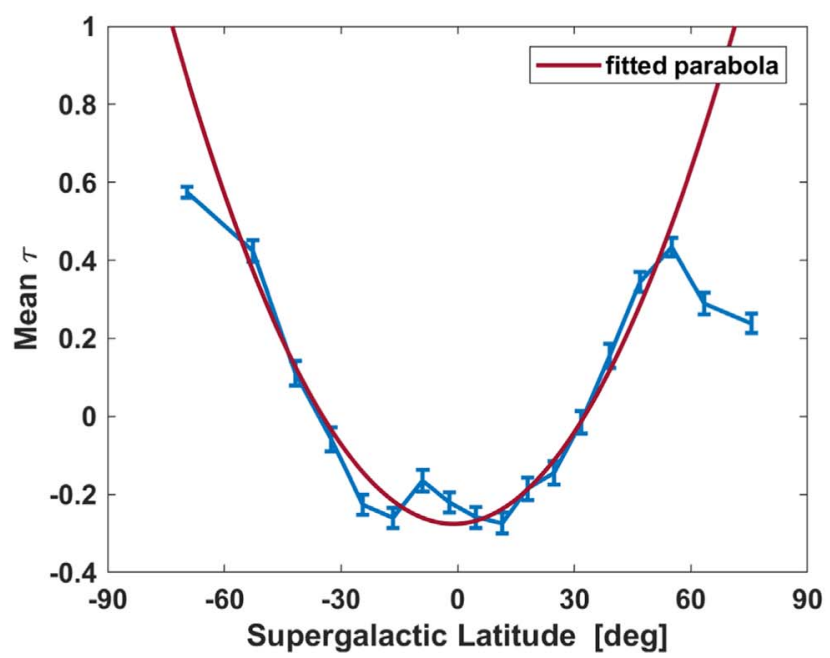

(b)

Figure 7. Seven year data result. (a) Projection of the correlation strength $\tau$ for all grid points. Negative correlations expected for magnetic deflections are apparent around the supergalactic plane. Solid curves indicate the galactic plane (GP) in blue and supergalactic plane (SGP) in red. White and gray hexagrams indicate the galactic center (GC) and antigalactic center (anti-GC), respectively. (b) Mean $\tau$ inside equal solid angle bins of supergalactic latitude (SGB). The correlation curvature is $a=(2.45 \pm 0.15) \times 10^{-4}$.

Figure 8(b) shows the mean $\tau$ correlation for 10 years of data with no new scan of wedge parameters for maximum correlation significances. The parabola curvature is $a=(1.60$ $\pm 0.09) \times 10^{-4}$, and the minimum is at $1.1 \mathrm{SGB}$. According to the $R^{2}=0.91$ goodness-of-fit, the model predicts $91 \%$ of the variance of the data. It can be seen that the correlations are similar to the seven year result, though the supergalactic structure may not be quite as significant.

\subsection{Significance of Supergalactic Structure}

By applying this analysis to isotropic MC sets, as described in Section 3.1, and counting the number of MC with an $a$ parameter larger than data (Figure 7(b)), the posttrial significance of the supergalactic structure of multiplets can be found. The resulting $a$ distribution of 1,000,000 MC sets is shown in Figure 9 for the seven year data statistics and energyangle correlation significance scan.

For the seven year data analysis, there are $14 \mathrm{MC}$ sets with a larger curvature than the data, which result in the significance of a supergalactic structure of multiplets of $\sim 4.2 \sigma$.

For the 10 years of data with no updated wedge correlation significance scan, the resulting $a$ distribution of 1,000,000 MC sets is shown in Figure 10. The distribution has a smaller standard deviation due to no new scans for energy-angle correlation significances. The result is a smaller $\tau$ on average. 


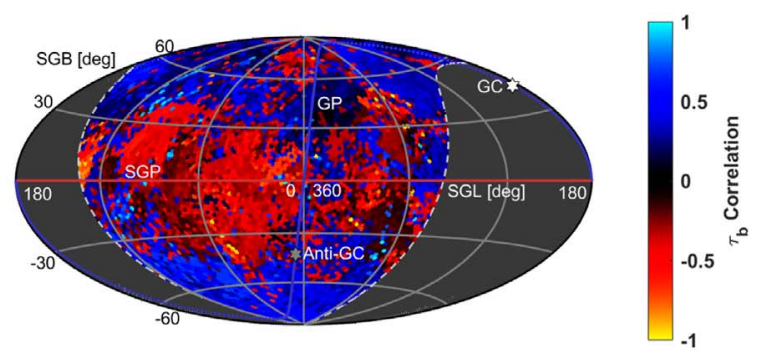

(a)

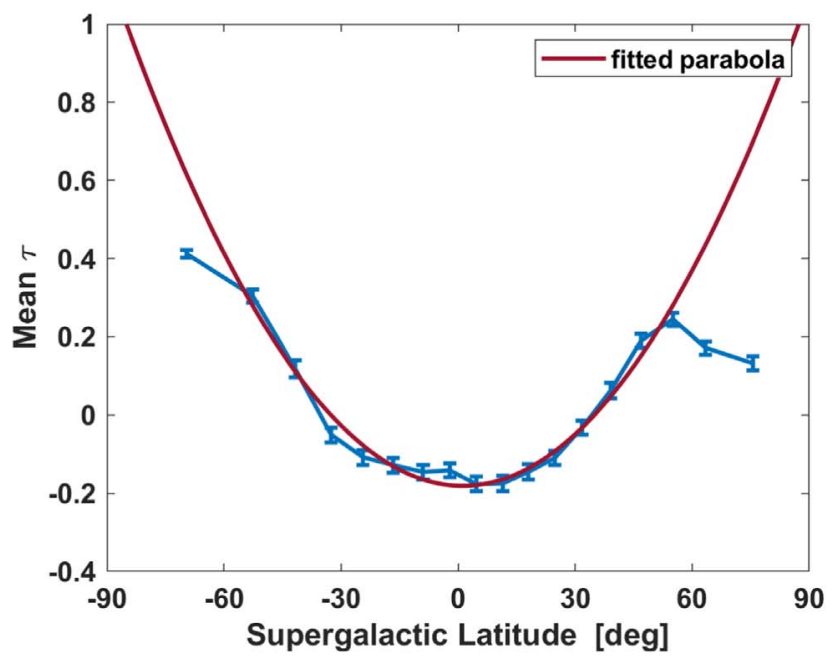

(b)

Figure 8. Ten years of data result. (a) Projection of the correlation strength $\tau$ for all grid points. Negative correlations expected for magnetic deflections are apparent around the supergalactic plane. (b) Mean $\tau$ inside equal solid angle bins of supergalactic latitude (SGB). The correlation curvature is $a=(1.60 \pm 0.09) \times 10^{-4}$.

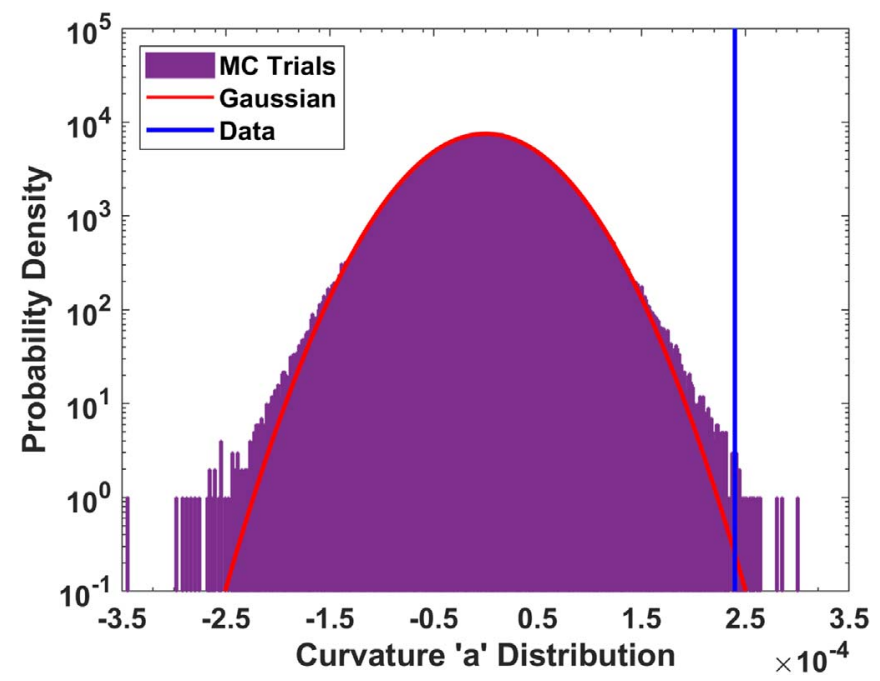

Figure 9. The distribution of the curvature parameter $a$ of the mean $\tau$ parabola chosen as the supergalactic structure of multiplets test statistic for 1,000,000 isotropic MC sets. The purple bars are the MC probability distribution function (PDF). The red line is a Gaussian distribution fit to the MC distribution. The curvature for the data is $a=2.45 \times 10^{-4}$ shown as a blue vertical line. There are $14 \mathrm{MC}$ with a larger curvature than the data, which gives a significance of $4.2 \sigma$.

There are $22 \mathrm{MC}$ sets with a larger curvature than the data, shown in Figure 8(b), which results in the significance of a supergalactic structure of multiplets of $\sim 4.1 \sigma$.

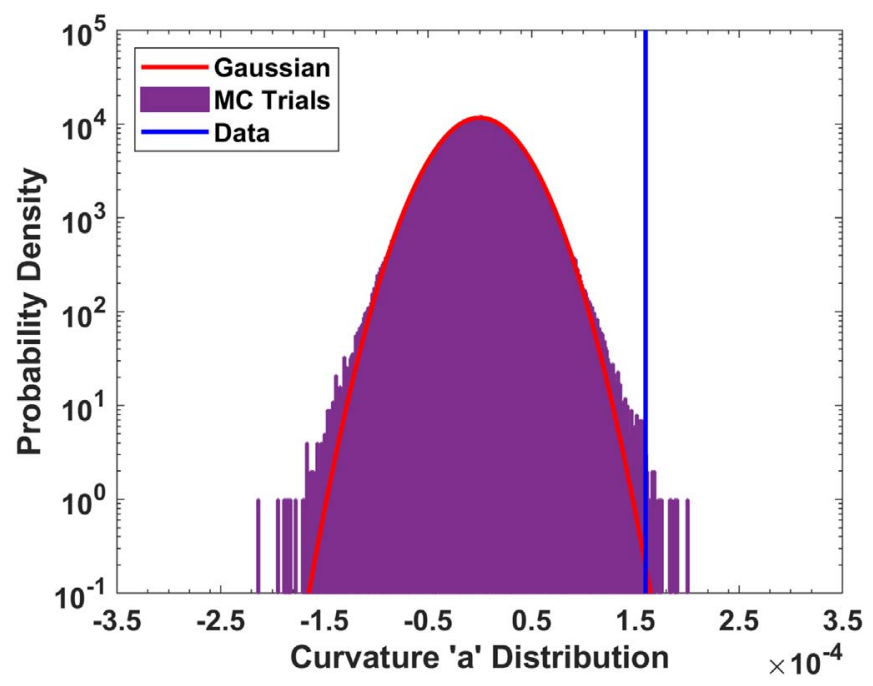

Figure 10. The distribution of the curvature parameter $a$ of the mean $\tau$ parabola chosen as the supergalactic structure of multiplets test statistic for 900,000 isotropic MC sets. The purple bars are the MC PDF. The red line is a Gaussian distribution fit to the MC distribution. The curvature for the data is $a=1.60 \times 10^{-4}$ shown as a blue vertical line. There are $22 \mathrm{MC}$ with a larger curvature than the data, which gives a significance of $4.1 \sigma$.

The total number of MC sets that were used to calculate the significance was limited by the computing time necessary for each simulation. Overall, the number of correlations calculated was $4 \times 10^{14}$, and this took more than 200 years of equivalent CPU computing time.

\subsection{Scan Parameter Distributions}

Clues about UHECR sources, and intervening fields, may be found from the maximum significance wedge scan parameters of the apparent magnetic deflection multiplets. Due to the significance maximization, there is a bias toward greater statistics, as can be seen in Equation (4), so the data are compared to isotropic MC by taking the ratio of the parameter probability distribution functions (PDFs; normalized histograms of data divided by MC). The PDF ratio shows how many times more likely a scan parameter value is to be found in data than isotropic MC. PDF ratio plots for wedge pointing direction and energy threshold parameters are shown in Figure 11.

These ratios are done for negative energy-angle correlations at grid-point positions $|\mathrm{SGB}| \leqslant 40^{\circ}$ (about the boundary where the average correlation is zero as shown in Figure 8(a)) and have a linear fit to $1 / E$ versus angular distance with $R^{2}>0$ (Figure $3(\mathrm{~b})$ ). An $R^{2}>0$ is a better fit than a horizontal line, and the $\delta \propto 1 / E$ model explains some of the variance of the data inside the wedge. For data, there are 2045 correlations used and greater than $3.99 \times 10^{8}$ for MC.

The data distribution of wedge pointing directions, Figure 11(a), provides further indication of a supergalactic structure with four deviations seemingly correlated with the supergalactic plane (SGP). Two larger peaks are approximately perpendicular to the SGP $\left(\sim 195^{\circ}\right.$ and $\left.\sim 345^{\circ}\right)$, and two smaller peaks are close to parallel $\left(\sim 90^{\circ}\right.$ and $\left.\sim 285^{\circ}\right)$. These peaks suggest an overall diffusion of low energy events away from the supergalactic plane, similar to the supergalactic magnetic sheet simulation of Section 3.2.

The data distribution of the energy threshold parameters may provide information regarding UHECR sources and intervening fields. The median energy threshold is $30 \mathrm{EeV}$, and the three 


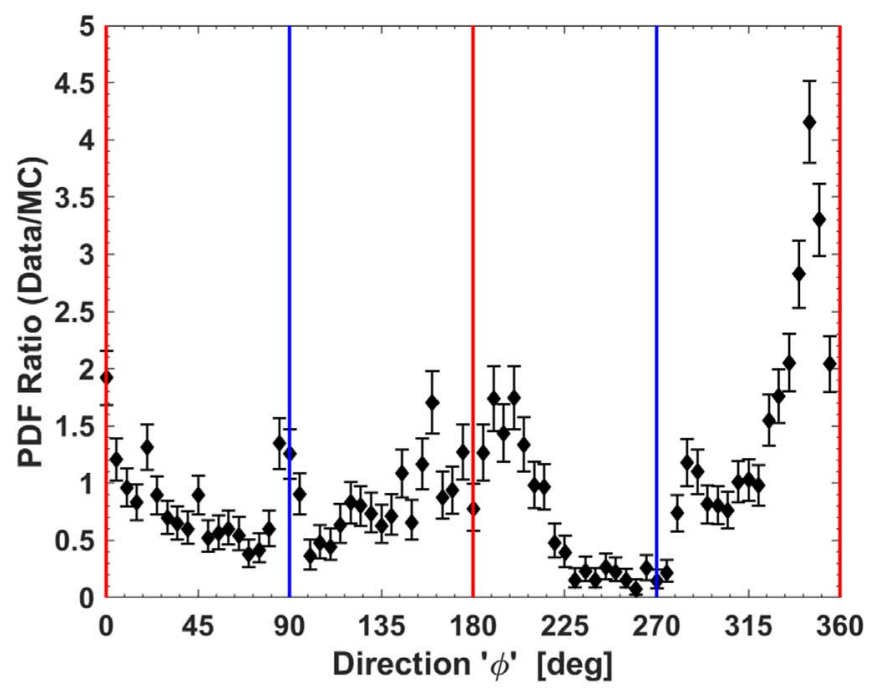

(a)

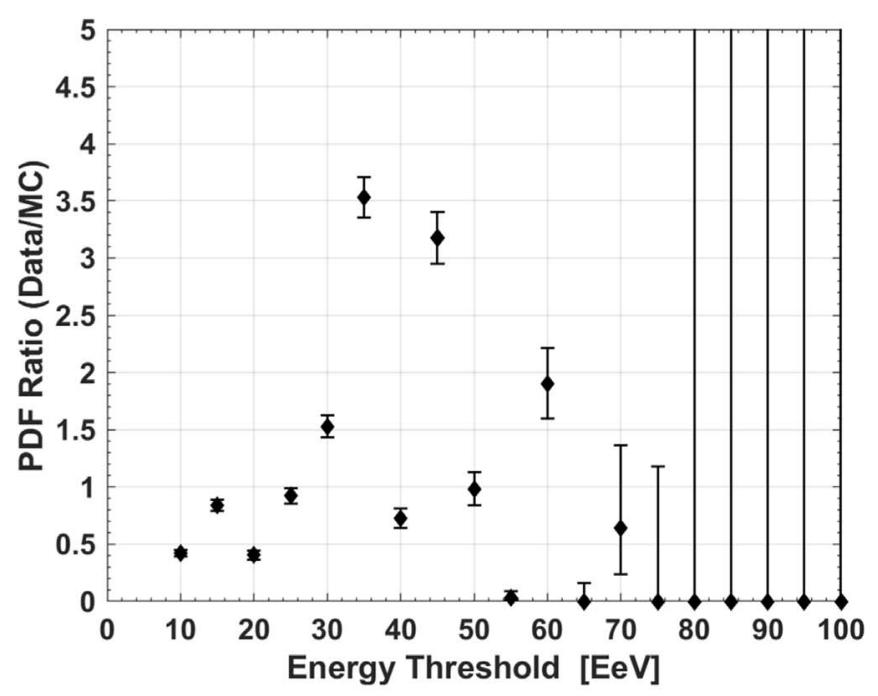

(b)

Figure 11. PDF ratio plot of scanned parameters. (a) Wedge pointing direction parameter, $\phi$. This distribution provides further indication of the supergalactic structure of multiplets. The blue vertical lines are parallel to the SGP. The red lines are perpendicular to the SGP. Two significant peaks can be seen nearly perpendicular, and two smaller peaks nearly parallel, to the SGP. (b) Energy threshold, E. The three largest deviations are at $35 \mathrm{EeV}, 45 \mathrm{EeV}$, and $60 \mathrm{EeV}$. This distribution may provide information regarding UHECR sources and intervening fields.

largest deviations from the isotropic distribution are at $35 \mathrm{EeV}$, $45 \mathrm{EeV}$, and $60 \mathrm{EeV}$. The $60 \mathrm{EeV}$ peak appears to correspond to the $57 \mathrm{EeV}$ threshold of the TA Hotspot analysis (Abbasi et al. 2014).

The median energy threshold of $30 \mathrm{EeV}$ is above the significant Pierre Auger Observatory (PAO) large-scale dipole measurements in Aab et al. (2018a) at $8 \mathrm{EeV}$, which is consistent with the localized intermediate-scale energy-angle magnetic deflections in this analysis.

The $39 \mathrm{EeV}$ cutoff for maximum event correlation with starburst galaxies, reported by PAO in Aab et al. (2018b), may be related to the $35 \mathrm{EeV}$ and $45 \mathrm{EeV}$ peaks.

These threshold deviations from isotropy are also consistent with the result using AGASA data that showed a possible large-scale cross-correlation between UHECR and the

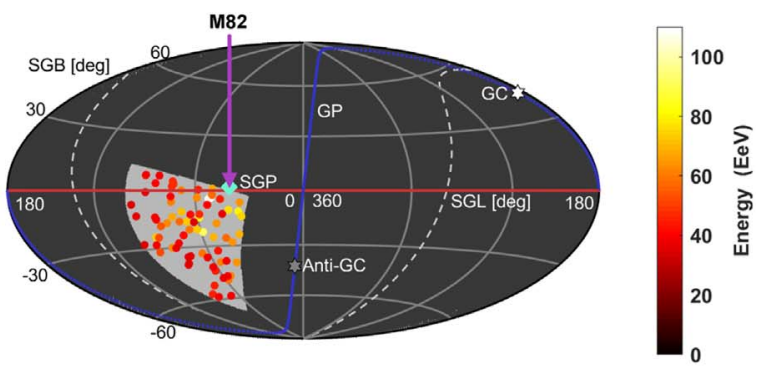

(a)

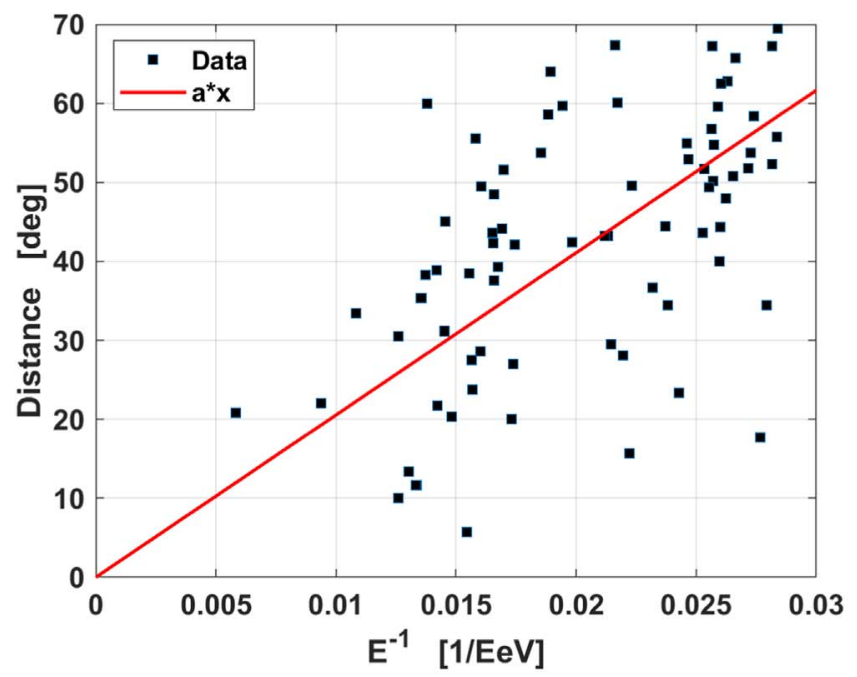

(b)

Figure 12. (a) Supergalactic projection of the most significant "wedge" multiplet of 10 years of data at $30^{\circ} 3 \mathrm{SGB},-3.2$ SGL. The correlation $\tau=-0.412$ with 75 data events has a pretrial one-sided significance of $5.10 \sigma$. This significance is an increase from $4.58 \sigma$ at this grid point, with seven years of data. The energy threshold is $E_{i} \geqslant 35 \mathrm{EeV}$, wedge width $W_{i}=90^{\circ}$, angular distance $D_{i}=70^{\circ}$, and direction $\phi_{i}=120^{\circ}$. The blue diamond is the location of the starburst galaxy M82. (b) Scatter plot of $1 / E_{j}$ vs. angular distance $\delta_{j}$ in the wedge. A linear fit (by Equation 1 (a) with $Z=1$ ) results in an estimate of $B \times S=41 \mathrm{nG} * \mathrm{Mpc}$. If the source is assumed to be at the same distance to M82 $(3.7 \mathrm{Mpc})$ with a pure proton emission, then the average coherent magnetic field required to cause this deflection would be $B=11 \mathrm{nG}$.

supergalactic plane between 50 and $80 \mathrm{EeV}$ energy bins (Burgett \& O'Malley 2003). Adjusting the AGASA energy scale to the TA energy scale by multiplying by 0.75 , this becomes 38 and $60 \mathrm{EeV}$ energy bins.

The data distributions of wedge angular distance, $D$, and width, $W$, do not show any significant deviations from isotropy.

\subsection{M82 Galaxy as Anisotropy Source}

The most significant single correlation using 10 years of SD data is at $30.3 \mathrm{SGB},-3.2 \mathrm{SGL}$, and shown in Figure 12(a). With 75 events $(\mathrm{E} \geqslant 35 \mathrm{EeV})$ and $\tau=-0.412$, it has a pretrial significance of $5.10 \sigma$. This significance is an increase from $4.58 \sigma$ at this grid point, with seven years of data using the same wedge and energy threshold parameters. Figure 12(b) shows a scatter plot of energy versus angular distance. A linear fit (Equation 1(a) with $Z=1$ ) results in an estimate of $B \times S=41$ $\mathrm{nG} * \mathrm{Mpc}$.

Recently, PAO has stated that the likeliest source of events with $E>39 \mathrm{EeV}$ are starburst galaxies (Aab et al. 2018b). The most significant correlation reported here is 11.3 from M82 (as 


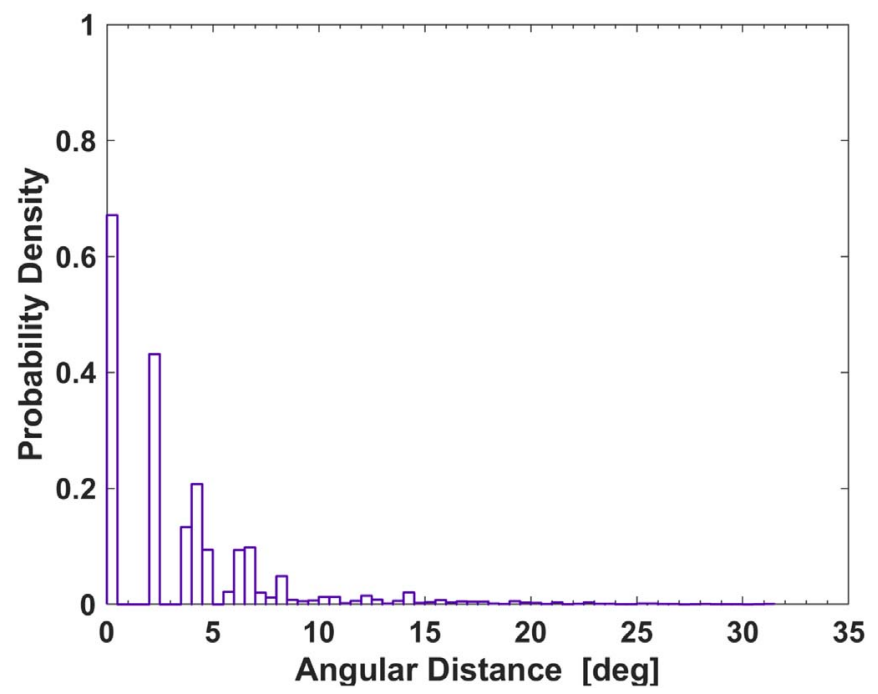

Figure 13. Distribution of distances from the actual most significant correlation grid point to those found in randomized data with the wedge embedded.

shown by the blue diamond in Figure 12(a)), pointing directly over the TA Hotspot (Figure 2). M82 is the closest starburst galaxy to our galaxy.

If the source is assumed to be at the same distance to M82 $(3.7 \mathrm{Mpc})$ with a pure proton emission, the average coherent magnetic field perpendicular to the FOV required to cause this deflection is $B=11 \mathrm{nG}$. The large deviations from the linear fit of Figure 12(b) imply that, in this region, the random field deflections have a large correlation length scale $\left(L_{c}\right)$, and the random field ( $B_{\text {rms }}$ ) of Equation 1(b) is on the same order of magnitude as the coherent field deflection.

Random variations of the data are created to estimate the uncertainty on the location of the source of the maximum significance energy-angle correlation. The energies of events outside the wedge are scrambled with other events outside the wedge. Inside, the energies of wedge events with energy less than the wedge threshold, $E<35 \mathrm{EeV}$, are randomized within the wedge. The locations of the 75 data events in the wedge with $E \geqslant 35 \mathrm{EeV}$ are not changed. This ensures that the spectrum is not changed, inside or outside the wedge, and that the number of events $E \geqslant 35 \mathrm{EeV}$ does not dramatically increase due to the Coldspot (Abbasi et al. 2018a). The analysis, including scanning for maximum significance wedge correlations at all grid points, was repeated for 5000 of these random variations on the data.

The estimated location of each randomized data set source is the most significant negative correlation near the known source grid point. The maximum distance searched, within a spherical cap centered on the known grid point, is the distance that minimizes the average $\tau$ inside the cap (correlations are more positive outside). A spherical cap limiter is necessary due to the fact that an entirely different set of events from the wedge of interest, say on the other side of the FOV, can easily have a more significant correlation due to the number of scans done at each grid point.

The result is that the apparent sources have a median distance of $2^{\circ} .4$ from the original source with a $+1 \sigma$ quantile of 6.8 and $6.2 \%$ are greater than or equal to 11.3 away (the angular distance from M82 to the maximum significance grid point). The distribution of distances is shown in Figure 13. This distribution means that M82 is not excluded as a possible

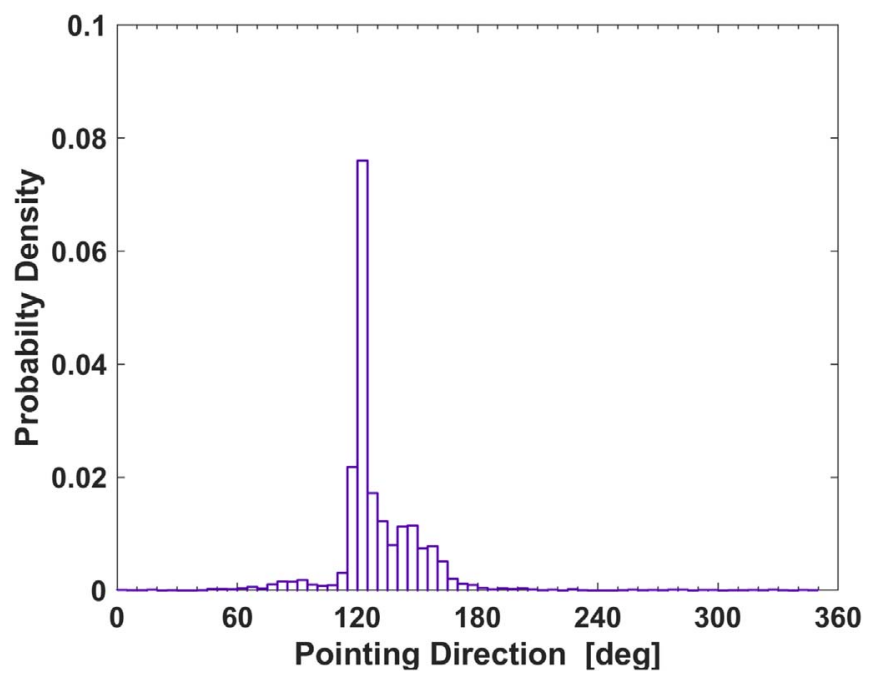

Figure 14. Distribution of the pointing direction of wedges found in randomized data with the most significant wedge embedded.

source of the events in this energy-angle correlation and the TA UHECR Hotspot/Coldspot (Abbasi et al. 2018a).

The result presented here appears to be consistent with the results of He et al. (2016), who used a Bayesian analysis of the relative deflection of TA Hotspot events in two energy bins $(E<75 \mathrm{EeV}$ and $E>75 \mathrm{EeV})$. Their result was a $99.8 \%$ probability that M82 is the Hotspot source.

According to the recent light polarization measurement of M82's magnetic field in Jones et al. (2019), the integrated magnetic field angle is $351^{\circ}$ in equatorial coordinates using the same definition as Section 2.2. Rotating into supergalactic coordinates results in an angle of $308^{\circ}$. The coherent magnetic field direction necessary to create the most significant multiplet is $120 \pm 90^{\circ}$, so it is either $82^{\circ}$ or $98^{\circ}$ from M82's magnetic field direction. The circular standard deviation of the pointing direction of the wedge simulations shown in Figure 14 is $21^{\circ}$, which means the wedge magnetic field direction is $\geqslant 4 \sigma$ different from M82's magnetic field direction. This direction discrepancy implies that if M82 is the source, then magnetic fields outside M82 were the primary source of multiplet pattern deflections.

\subsection{Supergalactic Field Estimate}

The average linear fit to $1 / E$ versus angular distance from the grid point, inside wedges with a negative correlation, can give an estimate of coherent magnetic field strength times the distance traveled through the field (see Equation 1(a) as shown in Figure 13(b)). These $B \times S$ values are independent of the ranked correlation pretrial significances, which were maximized to choose the wedge parameters.

If the coherent magnetic field in the vicinity of positive correlations is considered negligible and those correlations are set to $B \times S=0$, then the mean $B \times S$ in supergalactic latitude (SGB) bins appears as Figure 15. Given $\langle B \times S\rangle=$ $21 \mathrm{nG} * \mathrm{Mpc}$ and if the composition is protonic, then the average coherent field component, perpendicular to the FOV, in the vicinity of the supergalactic plane $\left(|\mathrm{SGB}| \leqslant 40^{\circ}\right)$ is $5.6 \mathrm{nG}$ (assuming a source distance of $3.7 \mathrm{Mpc}$ ).

If the coherent magnetic field in the vicinity of positive correlations is considered to be unknown, and those correlations are ignored, then the mean $B \times S$ in supergalactic latitude 


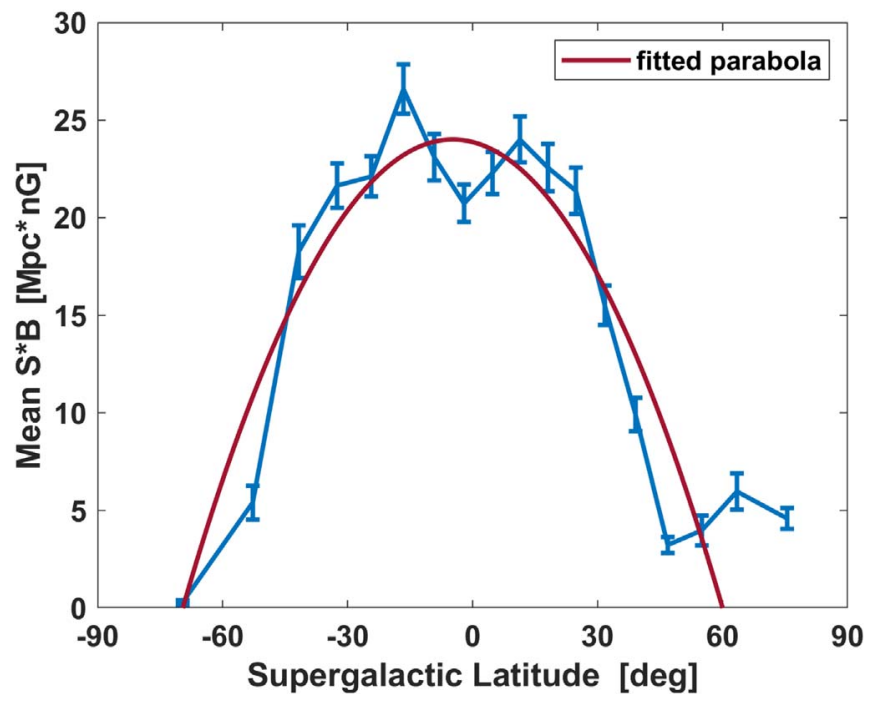

Figure 15. Mean $B \times S$ inside equal solid angle SGB bins setting $B \times S=0$ for wedges with positive correlations. The fitted parabola also demonstrates the correlation between apparent magnetic deflection multiplets with the supergalactic plane. These values are independent of the ranked correlation pretrial significances, which were maximized to choose the wedge parameters. The mean within $|\mathrm{SGB}| \leqslant 40^{\circ}$ is $\langle B \times S\rangle=21 \mathrm{nG} * \mathrm{Mpc}$. If proton is the assumed composition, then the average coherent field component, perpendicular to the $\mathrm{FOV}$, in the vicinity of the supergalactic plane assuming a distance of $3.7 \mathrm{Mpc}$ is $5.6 \mathrm{nG}$.

(SGB) bins appears as Figure 16. If proton is the assumed composition, then the average coherent field component, perpendicular to the FOV, in the vicinity of the supergalactic plane assuming a distance of $3.7 \mathrm{Mpc}$ is $\sim 8.6 \mathrm{nG}$.

Recently in Globus et al. (2019), the best-fit average extragalactic field to PAO dipole, assuming a local large-scale structure (LSS) distribution of sources according to the CosmicFlows-2 catalog, was estimated to be $0.6 \mathrm{nG}$ using PAO mixed composition $E \geqslant 8 \mathrm{EeV}$ in Aab et al. (2017). If the TA composition is largely protonic, as in Abbasi et al. (2018b), then the average distance traveled, $S$, necessary for agreement with $\mathrm{PAO}$ on the extragalactic field strength is $\sim 50 \mathrm{Mpc}$. The mean distance of galaxies in the CosmicFlows-2 catalog, within the GZK horizon of $\sim 100 \mathrm{Mpc}$, is $51 \mathrm{Mpc}$ (Tully et al. 2013). Given the model and experimental uncertainties, TA and PAO seem to have a good order of magnitude agreement on the extragalactic field strength.

\section{Systematic Checks}

A test of variation of isotropic MC parameters was done by calculating the significance of the supergalactic structure for seven years of data using two different MC. The first MC used the actual positions of the data and randomized energies according to the energy spectrum. This result had a $4.3 \pm 0.2 \sigma$ significance ( 2 out of 200,000 trials with an $a$ parameter greater than data) and was reported in Lundquist \& Sokolsky (2019). That significance is consistent with the current result, using a completely isotropic position MC of $4.2 \sigma$ with over five times more $\mathrm{MC}$ sets used in the calculation.

\subsection{Energy/Temperature Systematic}

Though ranked correlation is likely to decrease the effect of systematics, and temperature is taken into account for energy

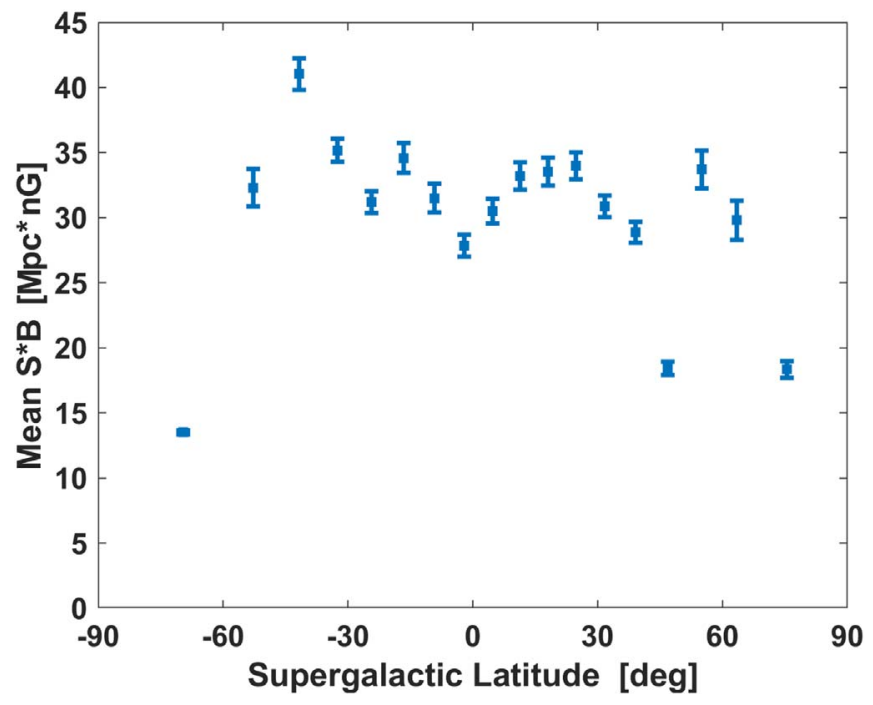

Figure 16. Mean $B \times S$ inside equal solid angle SGB bins not counting wedges with positive correlations. These values are independent of the ranked correlation pretrial significances, which were maximized to choose the wedge parameters. The mean within $|\mathrm{SGB}| \leqslant 40^{\circ}$ is $\langle B \times S\rangle=32 \mathrm{nG} * \mathrm{Mpc}$. If proton is the assumed composition, then the average coherent field component, perpendicular to the FOV, in the vicinity of the supergalactic plane assuming a distance of $3.7 \mathrm{Mpc}$ is $8.6 \mathrm{nG}$.

reconstruction, there is a possibility of a residual amount of correlation between the two. To test for this, each event trigger time was assigned the closest in time temperature measurement from three Delta, Utah stations taken from the NOAA databases (NOAA National Centers for Environmental Information 2019).

Using the 10 year data set, the correlation between energy and temperature is $\tau=0.027$ (a small tendency for energy to increase with increasing temperature) with a $0.9 \%$ probability it is actually zero given enough samples. Additionally, there may be a correlation between angular distance from a grid point, and temperature as the average temperature in equatorial R.A. varies about $5^{\circ}$.

To check the possibility that the supergalactic structure found could be an artifact of temperature variations, the partial Kendall correlation, $\tau_{x y . z}$, between energy and angular distance is done, removing temperature as a possible confounding variable. This is shown in Equation (7) ( $x$ stands for energy, $y$ for angular distance, and $z$ for temperature):

$$
\tau_{x y . z}=\frac{\tau_{x y}-\tau_{x z} \tau_{y z}}{\left(1-\tau_{x z}^{2}\right)\left(1-\tau_{y z}^{2}\right)} .
$$

The average $\tau_{x y . z}$ in equal solid angle bins of supergalactic latitude (SGB) results in a parabolic fit curvature decrease of $0.8 \%$. This decrease is a very small difference and likely an effect of noise in the temperature measurements used. Therefore, no evidence for a temperature anisotropy producing the results is found.

\subsection{Galactic Field Influence}

The energy-angle correlation wedge parameter space should minimize the number of exclusively galactic field created correlations that result from the correlation significance scan. The minimum wedge distance is $15^{\circ}$ (with a resulting mean of $63^{\circ}$ for 10 years of data), and the minimum wedge width is $10^{\circ}$ 


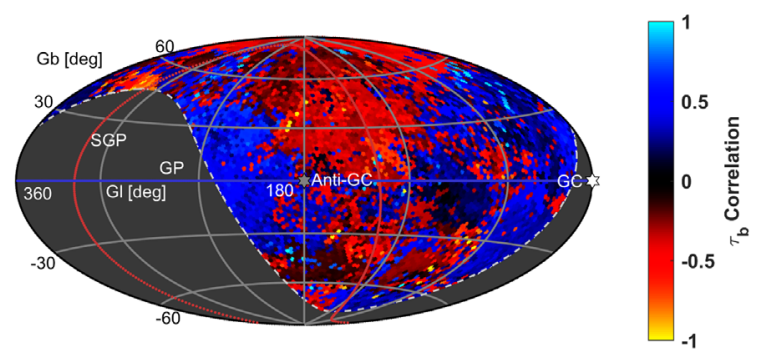

(a)

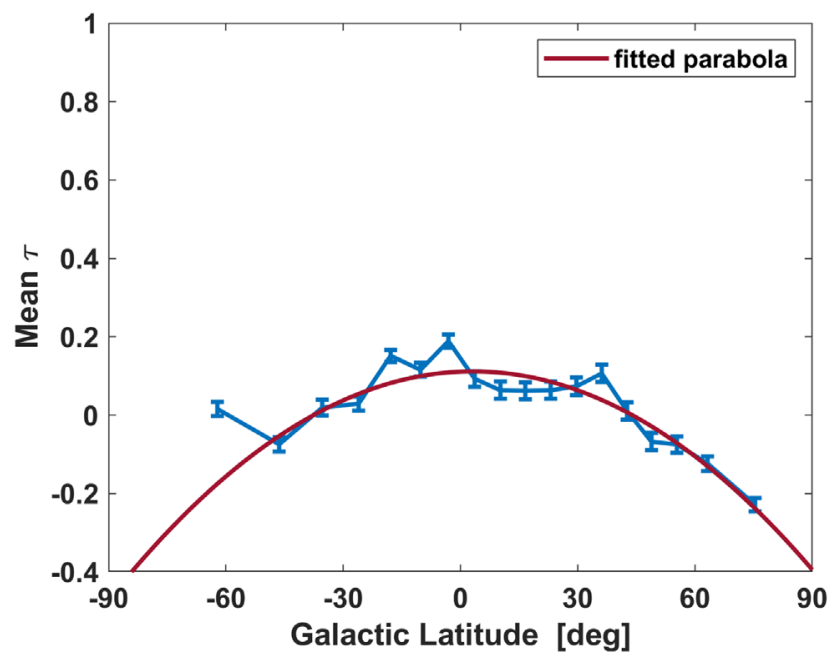

(b)

Figure 17. Ten years of data result shown in galactic coordinates. (a) HammerAitoff galactic projection of the correlation strength $\tau$ for all grid points. Negative correlations expected for magnetic deflections are not apparent around the galactic plane. (b) Mean $\tau$ inside equal solid angle bins of galactic latitude $(\mathrm{Gb})$. The resulting correlation structure curvature is $a=$ $-6.7 \times 10^{-5}$.

(with a mean of $26^{\circ}$ ). The mean galactic magnetic field deflection expectation for UHECR protons with energies $E \geqslant 26 \mathrm{EeV}$ (the average data wedge energy threshold) is $\leqslant \sim 15^{\circ}$ for the various models in Farrar \& Sutherland (2019), and the expected dispersion around the mean is $<10^{\circ}$ for $E \geqslant 10 \mathrm{EeV}$

The result of rotating into galactic coordinates and plotting the $\tau$ at each grid point for the 10 years of data is shown in Figure 17(a). The negative curvature of the average $\tau$ with respect to galactic latitude (Gb), shown in Figure 17(b), could suggest that possible magnetic deflections from apparent sources closer to the galactic plane are influenced by galactic magnetic fields with different directions from the average extragalactic fields. This behavior is consistent with the average widening of the wedge bins near the galactic plane shown in Figure 18.

Additionally, no apparent galactic structure of multiplets is found by the method in Section 6.1 when rotating the galactic coordinates by $90^{\circ}$. This is shown in Figure 19 by the average $\tau$ in equal solid angle bins of galactic longitude $(\mathrm{Gl})$ centered on the intersection between the galactic plane (GP) and the supergalactic plane (SGP). This rotation is where the correlations appear to have the most galactic symmetry according to Figure 17(a) though the resulting correlation curvature $a$ from the fit is $18 \%$ of the supergalactic curvature result.

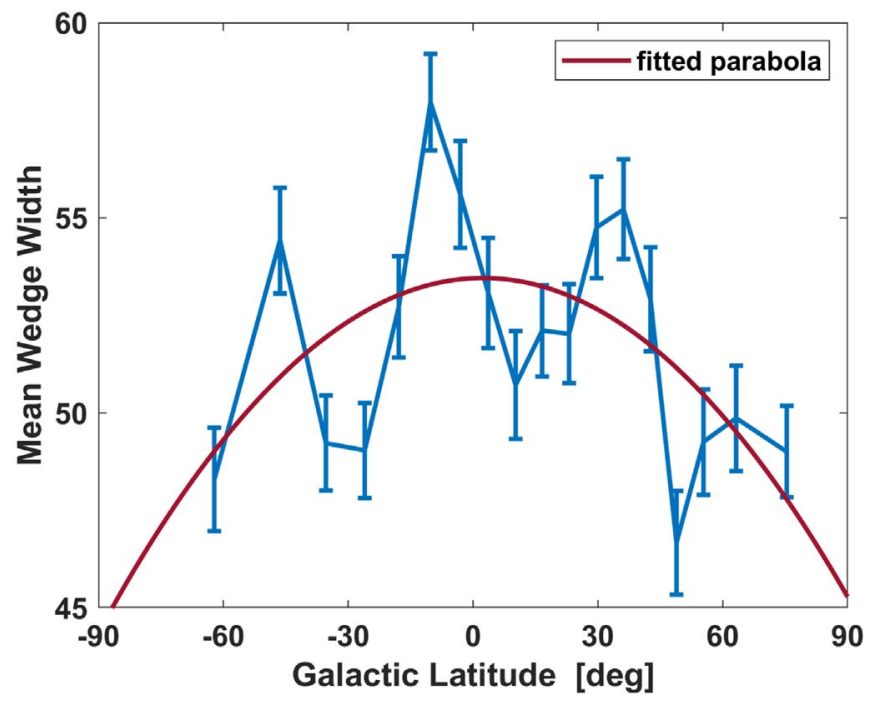

Figure 18. The mean wedge width inside equal solid angle bins of galactic latitude $(\mathrm{Gb})$ for the 10 years of data result. Wider bins are consistent with larger random field deflections near the galactic plane.

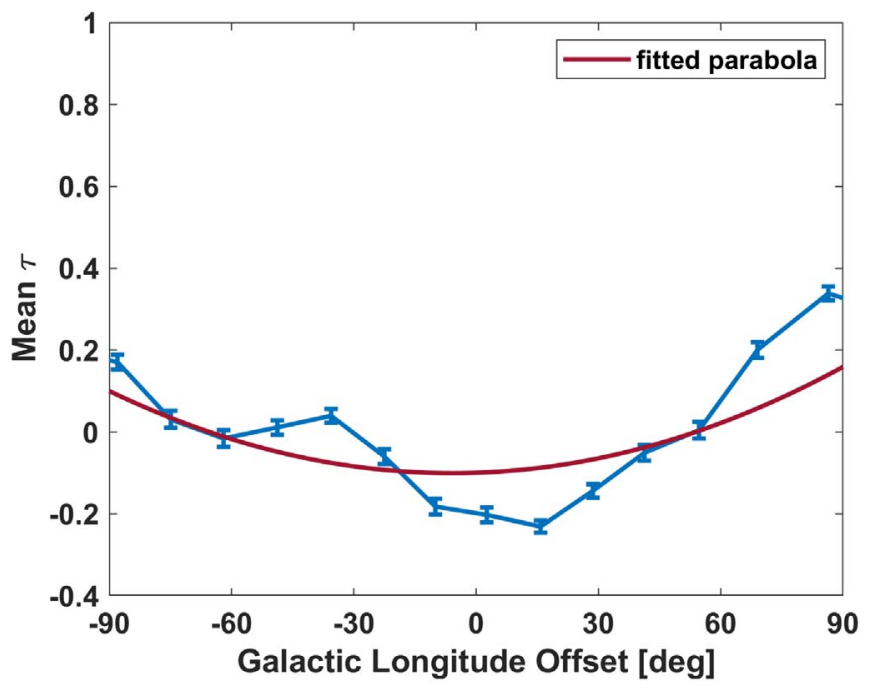

Figure 19. Ten years of data result shown in galactic coordinates for the mean $\tau$ inside equal solid angle bins of galactic longitude (Gl) centered on the intersection between the galactic plane (GP) and the supergalactic plane (SGP).

\section{Summary}

Intermediate-scale energy-angle correlations inside spherical cap sections, or "wedges," have been shown to have a $\sim 4 \sigma$ correlation with the supergalactic plane. Seven years of TA data have a $4.2 \sigma$ posttrial significance, and the 10 years of data significance is $4.1 \sigma$ posttrial. These results may be evidence of large-scale extragalactic magnetic diffusion of UHECR from sources within the local LSS as there does not appear to be a galactic correlation structure.

Additionally, the highest significance single energy-angle correlation has increased from a pretrial $4.6 \sigma$ significance (in the 7 years of data) to $5.1 \sigma$ (in the 10 years of data) with no new scan of wedge parameters. This correlation lies directly over the TA Hotspot, and its origin point is consistent with the starburst galaxy M82 being a source of these events. This result is consistent with other results assuming magnetic deflection such as $\mathrm{He}$ et al. (2016) and with the starburst galaxy overdensity anisotropy study of Aab et al. (2018b). 
If M82 is the source of the most significant correlation, then the average coherent magnetic field component perpendicular to the FOV, within this section of the sky, is estimated to be 11 nG assuming a purely proton composition.

The average perpendicular magnetic field correlated with the supergalactic plane is also estimated to be on the order of 10 nG assuming a cosmic-ray travel length of $3.7 \mathrm{Mpc}$ and a proton composition. A mixed composition and/or a longer travel length results in a smaller magnetic field estimate. This result is consistent with other estimates of extragalactic magnetic fields via theory, simulation, and astrophysical measurements (Ryu et al. 1998; Globus et al. 2019; Kronberg et al. 1994 for example).

Confirmation of these results awaits sufficient data to be collected by the TA expansion to TAx4 (Kido 2019).

The Telescope Array experiment is supported by the Japan Society for the Promotion of Science (JSPS) through Grants-inAid for Priority Area 431, Specially Promoted Research JP21000002, Scientific Research (S) JP19104006, Specially Promoted Research JP15H05693, Scientific Research (S) JP15H05741, Science Research (A) JP18H03705, and for Young Scientists (A) JPH26707011; by the joint research program of the Institute for Cosmic Ray Research (ICRR), The University of Tokyo; by the U.S. National Science Foundation awards PHY-0601915, PHY-1404495, PHY-1404502, and PHY-1607727; by the National Research Foundation of Korea (2016R1A2B4014967, 2016R1A5A1013277, 2017K1 A4A3015188, 2017R1A2A1A05071429); by the Russian Academy of Sciences, RFBR grant 20-02-00625a (INR), IISN project No. 4.4502.13, and Belgian Science Policy under IUAP VII/37 (ULB). The foundations of Dr. Ezekiel R. and Edna Wattis Dumke, Willard L. Eccles, and George S. and Dolores Doré Eccles all helped with generous donations. The state of Utah supported the project through its Economic Development Board, and the University of Utah through the Office of the Vice President for Research. The experimental site became available through the cooperation of the Utah School and Institutional Trust Lands Administration (SITLA), US Bureau of Land Management (BLM), and the US Air Force. We appreciate the assistance of the State of Utah and Fillmore offices of the BLM in crafting the plan of development for the site. Patrick Shea assisted the collaboration with valuable advice on a variety of topics. The people and the officials of Millard County, Utah, have been a source of steadfast and warm support for our work which we greatly appreciate. We are indebted to the Millard County Road Department for their efforts to maintain and clear the roads which get us to our sites. We gratefully acknowledge the contribution from the technical staffs of our home institutions. An allocation of computer time from the Center for High Performance Computing at the University of Utah is gratefully acknowledged.

\section{ORCID iDs}

R. U. Abbasi (i) https://orcid.org/0000-0001-6141-4205

T. Abu-Zayyad (1) https://orcid.org/0000-0001-5206-4223

A. di Matteo (1) https://orcid.org/0000-0002-8260-1867

T. Fujii (1) https://orcid.org/0000-0003-2401-504X

W. Hanlon (1) https://orcid.org/0000-0002-0109-4737

D. Ikeda (1) https://orcid.org/0000-0003-1382-9267

D. Ivanov (1) https://orcid.org/0000-0002-4420-2830

C. C. H. Jui iㅏ https://orcid.org/0000-0002-1902-3478

J. P. Lundquist (1) https://orcid.org/0000-0002-4245-5092

J. N. Matthews (1) https://orcid.org/0000-0001-6940-5637

G. Rubtsov (1) https://orcid.org/0000-0002-6106-2673

Y. Tameda (ib https://orcid.org/0000-0001-9750-5440

S. Troitsky (i) https://orcid.org/0000-0001-6917-6600

Y. Tsunesada (1) https://orcid.org/0000-0001-9238-6817

\section{References}

Aab, A., Abreu, P., Aglietta, M., et al. 2015, EPJC, 75, 269

Aab, A., Abreu, P., Aglietta, M., et al. 2017, arXiv:1708.06592

Aab, A., Abreu, P., Aglietta, M., et al. 2018a, ApJ, 868, 4

Aab, A., Abreu, P., Aglietta, M., et al. 2018b, ApJL, 853, L29

Abbasi, R. U., Abe, M., Abu-Zayyad, T., et al. 2014, ApJL, 790, L21

Abbasi, R. U., Abe, M., Abu-Zayyad, T., et al. 2015, APh, 68, 27

Abbasi, R. U., Abe, M., Abu-Zayyad, T., et al. 2018a, ApJ, 862, 91

Abbasi, R. U., Abe, M., Abu-Zayyad, T., et al. 2018b, ApJ, 858, 76

Abbasi, R. U., Abu-Zayyad, T., Allen, M., et al. 2008, PhRvL, 100, 101101

Abreu, P., Aglietta, M., Ahn, E. J., et al. 2012, APh, 35, 354

Abu-Zayyad, T., Aida, R., Allen, M., et al. 2013, ApJL, 768, L1

Biermann, P. L., Kang, H., \& Ryu, D. 1997, arXiv:astro-ph/9709250

Bonafede, A., Feretti, L., Murgia, M., et al. 2010, A\&A, 513, A30

Bretz, H. P. 2011, PhD Thesis, Rheinisch-Westphalian Technical Univ. Aachen, http://www.migstd9.rz.rwth-aachen.de/fileadmin/user_upload/ www_physik/Personen/erdmann/Diplomathesis-Bretz.pdf

Burgett, W. S., \& O'Malley, M. R. 2003, PhRvD, 67, 092002

Croux, C., \& Dehon, C. 2010, Stat. Methods \& Applications, 19, 497

de Vaucouleurs, G. 1975, ApJ, 202, 610

Farrar, G. R., \& Sutherland, M. S. 2019, JCAP, 2019, 004

Globus, N., Ding, C., \& Farrar, G. R. 2019, ICRC, 36, 243

Govoni, F., Orrù, E., Bonafede, A., et al. 2019, Sci, 364, 981

He, H.-N., Kusenko, A., Nagataki, S., et al. 2016, PhRvD, 93, 043011

Ivanov, D. 2012, PhD Thesis, Rutgers Univ., http://telescopearray.com/ images/papers/theses/thesis_ivanov_rev2016.pdf

Jones, T. J., Dowell, C. D., Rodriguez, E. L., et al. 2019, ApJL, 870, L9

Kendall, M. G. 1945, Biometrika, 33, 239

Kido, E. 2019, EPJ Web Conf., 210, 06001

Kronberg, P. P. 1994, RPPh, 57, 325

Lundquist, J. P., \& Sokolsky, P. V. 2019, EPJ Web Conf., 210, 01006

Nicastro, F., Kaastra, J., Krongold, Y., et al. 2018, Natur, 558, 406

NOAA National Centers for Environmental Information 2019, Global Surface Hourly 2008-2015 Stations WBAN:53165, WBAN:23162, WBAN:00481, (Asheville, NC: NOAA National Centers for Environmental Information), https://www.ncdc.noaa.gov/cdo-web/datatools/lcd

Roulet, E. 2004, IJMPA, A19, 1133

Ryu, D., Kang, H., \& Biermann, P. L. 1998, A\&A, 335, 19

Teanby, N. A. 2006, CG, 32, 1442

Tully, R. B., Courtois, H. M., Dolphin, A. E., et al. 2013, AJ, 146, 86

Vallado, D. A. 2007, Fundamentals of Astrodynamics and Applications (Berlin: Springer)

Wirtz, M. 2019, ICRC, 36, 469 